CTP-SCU/2015021

\title{
Higher Spins at the Quintic Order: Localization Effect and Simplifications
}

\author{
Dimitri Polyakov ${ }^{\dagger(1),(2)}$ \\ Center for Theoretical Physics (1) \\ College of Physical Science and Technology \\ Sichuan University, Chengdu 610064, China \\ Institute for Information Transmission Problems $(\text { IITP })^{(2)}$ \\ Bolshoi Karetny per. 19/1 \\ 127994 Moscow, Russia
}

\begin{abstract}
We investigate the special case of quintic interactions for massless higher spin gauge fields using the string-theoretic vertex operator construction for higher spin gauge fields in Vasiliev's frame-like formalism. We compute explicitly the related 5-point interaction vertex in the low energy limit of string theory and find that: the structure of the quintic $s_{1}-s_{2}-s_{3}-s_{4}-s_{5}$ higher spin interaction gets drastically simplified and localized if

a) the spin values satisfy the constraint $s_{1}+s_{2}+s_{3}=s_{4}+s_{5}+2$ (and, more generally, if the sum of three spin values roughly equals the sum of the remaining two

b) One of the spin values, $s_{4}$ or $s_{5}$ is sufficiently small. In this paper, the explicit computation is done for the case $s_{4}=4$
\end{abstract}

November 2015

$\dagger(1),(2)$ polyakov@sogang.ac.kr ; polyakov@scu.edu.cn; twistorstring@gmail.com 


\section{Introduction}

Interacting higher-spin gauge fields are known to be the crucial ingredient of AdS/CFT and holography in general and, at the same time, difficult and fascinating objects to work with. Despite the fact that the higher spin theories in $A d S$ spaces can circumvent the restrictions imposed by the Coleman-Mandula's theorem, describing the gauge-invariant higher-spin interactions is a highly nontrivial problem since the gauge symmetry in these theories must be sufficiently powerful in order to eliminate unphysical degrees of freedom

[1], [2], [3], [4], [5], [6], [7], [8], [9], [10], [11], [12], [13], [14], [15], [16], [17], [18], [19], [20], [21], [22], [10], [22], [24], [25], [26], [27], [28], [29], [30], [31], [32], [33], [34], [30], [32], [27], [35], 36], 337, [38], [39], 440], [41], [42], [43]

The restrictions imposed by such a gauge symmetry make understanding the interactions in higher-spin theories a distinctively complicated problem. While there was some progress in classification of the higher-spin 3-vertices and cubic interactions over recent years, our understandingof the higher-order interactions is still largely incomplete even at the quartic level (with the quartic interactions presumably related to conformal blocks in the dual CFT's). Apart from that, at this point we know very little, if anything about higher spin theories beyond the quartic order, e.g. about quintic and higher order interactions. One general property expected from the interacting higher spin theories, is that they have to be essentially nonlocal. Such a nonlocality is a natural compromise in order to evade the restrictions imposed by Coleman-Mandula's theorem in flat space-time; however, it also appears to persist in curved background geometries such as AdS where, at least formally, the Coleman-Mandula's restrictions can be circumvented. In fact, this nonlocality property can be seen in a very natural way in string theory, where one interprets the higher-spin fields as space-time wavefunctions for the certain class of vertex operators and the higher spin interactions in terms of the worldsheet correlators of these operators. Indeed, string theory appears to be a natural framework to describe higher spin dynamics, as the frame-like description of the higher spin modes in Vasiliev's formalism has a very natural vertex operator interpretation [44]. The vertex operators describing higher spin modes have, however, the ghost structure which is very different from that of operators for the lower spin modes, such as a photon [44], [45] First of all, these operators couple nontrivially to the $\beta-\gamma$ system of superconformal ghosts. These couplings are classified by the minimum superconformal ghost pictures carried by the operators. That is, the vertex operators of spin $s$ are the elements of ghost cohomologies $H_{-s} \sim H_{s-2}(s>2)$ implying that they are annihilated by direct picture changing transformation at negative 
picture $-s$ and by inverse picture changing transformation at dual positive picture $s-2$. Moreover, these operators also have an anomalous $b-c$ ghost number couplings, which is closely related to the nonlocalities in the resulting interactions. Namely, the standard $b-c$ pictures for lower spin vertices, such as a photon, involve either integrated form at ghost number 0 , or unintegrated form at ghost number 1 . Combined with $b-c$ ghost number anomaly cancellation condition, this requires 3 unintegrated operators and $N-3$ integrated,leading, for example, to the standard form of Veneziano amplitude, defining local quartic interaction terms in the low-energy effective action. The ghost structure of the higher-spin operators is different. Since these operators violate picture equivalence and have no picture 0 representation, one needs operators both at positive and negative superconformal ghost pictures to ensure the correct superconformal ghost number balance in correlators. The higher spin vertex operators at positive pictures, however, only exist in the integrated form, given by the integrals of three types of terms. That is, for spin $s$ operators these types carry the ghost structures $e^{(s-2) \phi}, c e^{(s-3) \phi+\chi}$ and $\partial c c e^{(s-4) \phi+2 \chi}$ respectively (where $\phi$ and $\chi$ are the bosonized superconformal ghosts). Typically, it is the first and the second types of terms that contribute to higher spin correlators. The second type, while being integrated operators, also carry the $b-c$ ghost number one. This leads to the possibility of the $b-c$ ghost number balance being saturated while having an extra integration in the correlator (compared to the correlators of vertices for lower spins). This extra integration leads to appearance of additional singularities in the resulting scattering amplitude. Unlike the poles of lower spin amplitudes (such as Veneziano amplitude), corresponding to particle exchanges, the extra singularities reflect the nonlocalities in the related interaction terms for higher spin modes in the low-energy effective action. Typically, the combination of superconformal and $b-c$ ghost number balance constraints dictates that the type 2 operators always contribute to the higher spin amplitudes, leading to nonlocalities in space-time (e.g. considered in [46]). However, as we point out in this work, there exists a class of amplitudes contributed by the first type operators only. These amplitudes are in turn related to the appearance of local gauge-invariant interactions for higher spins in the low-energy effective action. We find that the higher spin interaction vertices of this type:

1) only appear at higher orders of interaction, starting from the quintic order. The interactions of this type are absent at lower orders, such as the quartic order. 
2) only may appear if the spin values satisfy the localization constraint, that is, for the order $N$ higher spin interaction the sum of 3 spins must be roughly equal to the sum of the remaining $N-3$, i.e.

$$
s_{1}+s_{2}+s_{3}=s_{4}+\ldots+s_{N}+\alpha
$$

where $\alpha$ is of the order of 1 (in the concrete example of the quintic interaction, considered in this work, $\alpha=2$ ). The rest of this paper is organized as follows. In the next section, we review the vertex operator formalism for frame-like higher spin gauge fields of arbitrary spin values and perform a general analysis of derivative structure of higher spin interaction, related to the correlators of these operators. In the Section 3, we calculate the particular example of the 5-point amplitude, satisfying the constraint (1), leading to the localization effect in the quintics. The structure of the amplitude and of the interaction particularly simplifies if one of the spins, $s_{4}$ or $s_{5}$ has a relatively small value, up to 6 (in this work we consider the case $s_{4}=4$, with all other spin values arbitrary, up to localization constraint (1)). In the calculation, we particularly use the OPE formalism for the Bell polynomial operators, developed in the previous work 477 In the concluding section, we discuss the physical implications of the calculation done in this work and its possible generalizations.

\section{Frame-like Fields and Vertex Operators:}

\section{Review of the Formalism and Preliminary Derivative Analysis}

In the frame-like formalism a symmetric higher spin field of spin $s$ is described by the set of of $s$ two-row gauge fields $\Omega^{s-1 \mid t}(x) \equiv \Omega_{m}^{a_{1} \ldots a_{s-1} \mid b_{1} \ldots b_{t}}(x)(0 \leq t \leq s-1)$ appearing in the higher spin extension of Cartan's 1-form by generators of infinite-dimensional higher spin algebra:

$$
\Omega^{(1)} \equiv \Omega_{m} d x^{m}=\left(e_{m}^{a} T_{a}+\omega_{m}^{a_{1} a_{2}} T_{a_{1} a_{2}}+\sum_{s=3}^{\infty} \sum_{t=0}^{s-1} \Omega_{m}^{a_{1} \ldots a_{s-1} \mid b_{1} \ldots b_{t}} T_{a_{1} \ldots a_{s-1} \mid b_{1} \ldots b_{t}}\right) d x^{m}
$$

where $T_{a}, T_{a_{1} a_{2}}$ are the space-time isometry generators, $e$ and $\omega$ are the vielbeins and spin connections for $s=2$ and $T_{a_{1} \ldots a_{s-1} \mid b_{1} \ldots b_{t}}$ are the generators of the higher-spin algebra that envelops the space-time isometry algebra. In this formalism, only $t=0$ fields are genuinely dynamical and are related to Fronsdal's metric-type higher spin fields upon symmetrization. The fields with $t \neq 0$ are the extra fields, related to the Fronsdal's field $\Omega^{s-1 \mid 0}$ by generalized zero torsion constraints according to

$$
\Omega^{s-1 \mid t}(x) \sim \partial^{t} \Omega^{s-1 \mid 0}
$$


In curved backgrounds, such as AdS, the frame-like approach to higher spin dynamics is remarkably efficient. At the same time, string theory is known to be a particularly natural framework to describe the interactions of higher spin fields in terms of the worldsheet correlators of the vertex operators. In particular, the bosonic string spectrum in the tensionless limit contains vertex operators that can be interpreted as sourses for Fronsdal's higher spin modes, although the correlators calculated in this limit are somewhat difficult to interpret in terms of higher spin interactions in the field-theoretic low-energy limit of string theory [2], [3], [4], [5], [30], 31]. Apart from the tensionless limit, the vertex operators describing massless higher spin modes can be constructed in RNS superstring theory. These operators are the elements of nonzero ghost cohomologies $H_{-s} \sim H_{s-2}$, existing either at minimal negative picture $s$ and below, or dual minimum positive picture $s-2$ and above. These operators describe massless fields of spin $s \geq 3$ in the frame - like formalism (except for $s=3$ where the corresponding higher spin mode is actually a Fronsdal's field). BRST invariance constraints on these operators entail the on-shell constraints on the higher spin fields (such as Pauli-Fierz constraints) while BRST nontriviality conditions entail the gauge and diffeomorphism transformations. As these transformations shift the vertex operators by BRST-exact terms, the resulting correlation functions are gauge-invariant by construction. For $s=3$, the manifest expressions for the vertex operators in RNS superstring theory are rather simple and are given by 448, 44, 45]

$$
V_{2 \mid 0}(p, z)=\Omega_{m}^{a b}(p) c e^{-3 \phi} \psi^{m} \partial X_{a} \partial X_{b} e^{i p X}(z)
$$

at unintegrated minimal negative ghost picture -3 and

$$
V_{2 \mid 0}(p, z)=\Omega_{m}^{a b}(p) K \circ \int d z e^{\phi} \psi^{m} \partial X_{a} \partial X_{b} e^{i p X}(z)
$$

at minimal positive picture +1 which is always integrated. Here $X^{m}$ are the target space coordinates, $\psi^{m}$ are the RNS fermions and $K$ is the homotopy transform, necessary to ensure the BRST invariance of the positive picture operator (5). (see [48, 444, 445] for the detailed description of the transform).

The operators (4), (5) are thus the elements of $H_{-3} \circ H_{1}$.

The manifest expressions for the vertex operators $V_{s-1 \mid t}$ for the frame-like fields with $s>3$ become far more complicated, however, a significant simplification occurs for the 
case $t=s-3$. In this case, the explicit expressions for the vertex operators are given by 45

$$
V_{s-1 \mid s-3}^{(-)}=c e^{-s \phi} \partial X_{m_{1}} \ldots \partial X_{s-1} \psi^{\alpha_{0}} \partial \psi_{\alpha_{1}} \partial^{2} \psi_{\alpha_{2}} \ldots \partial^{s-3} \psi_{\alpha_{s-3}} e^{i p X} \Omega_{\alpha_{0}}^{m_{1} \ldots m_{s-1} \mid \alpha_{1} \ldots \alpha_{s-3}}(p)
$$

at the minimal negative unintegrated picture and

$$
\begin{array}{r}
V_{s-1 \mid s-3}^{(+)}=K \circ \int d z e^{s-2} \partial X_{m_{1}} \ldots \partial X_{s-1} \psi^{\alpha_{0}} \partial \psi_{\alpha_{1}} \partial^{2} \psi_{\alpha_{2} \ldots \partial^{s-3}} \psi_{\alpha_{s-3}} e^{i p X} \\
\times \Omega_{\alpha_{0}}^{m_{1} \ldots m_{s-1} \mid \alpha_{1} \ldots \alpha_{s-3}}(p)
\end{array}
$$

at the minimal positive picture representation with the result of the homotopy Ktransformation given explicitly by

$$
\begin{array}{r}
K \circ \oint d z e^{s-2} \partial X_{m_{1}} \ldots \partial X_{m_{s-1}} \psi^{\alpha_{0}} \partial \psi_{\alpha_{1}} \partial^{2} \psi_{\alpha_{2}} \ldots \partial^{s-3} \\
\psi_{\alpha_{s-3}} e^{i p X} \Omega_{\alpha_{0}}^{m_{1} \ldots m_{s-1} \mid \alpha_{1} \ldots \alpha_{s-3}}(p) \\
=A_{0}(p ; u)+A_{1}(p ; u)+A_{2}(p ; u)
\end{array}
$$

where

$$
\begin{aligned}
& A_{0}(p ; u)=\Omega_{\alpha_{0}}^{m_{1} \ldots m_{s-1} \mid \alpha_{1} \ldots \alpha_{s-3}}(p) \\
& \times \oint d z(z-u)^{2 s-4} B_{2 \phi-2 \chi-\sigma}^{(2 s-4)} e^{(s-2) \phi} \psi^{\alpha_{0}} \partial \psi_{\alpha_{1}} \partial^{2} \psi_{\alpha_{2} \ldots \partial^{s-3}} \psi_{\alpha_{s-3}} e^{i p X}(u) \\
& A_{1}(p ; u)=2 \Omega_{\alpha_{0}}^{m_{1} \ldots m_{s-1} \mid \alpha_{1} \ldots \alpha_{s-3}}(p) \oint d z(z-u)^{2 s-4} c e^{\chi+(s-3) \phi+i p X} \\
& \times\left\{\sum _ { k = 0 } ^ { s - 3 } ( - 1 ) ^ { k + 1 } k ! \left[\sum_{j=0}^{s-2+k} B_{\phi-\chi}^{(s+k-1)} \partial X_{m_{1}} \ldots \partial X_{m_{s-1}} \psi^{\alpha_{0}}\right.\right. \\
& \times \partial \psi_{\alpha_{1} \ldots \partial^{k-1}} \psi_{\alpha_{k-1}}\left(-i p_{\alpha_{k}}\right) \partial^{k+1} \psi_{\alpha_{k+1}} \ldots \partial^{s-3} \psi_{\alpha_{s-3}} \\
& +\sum_{j}^{s-2+k} \frac{1}{j !} B_{\phi-\chi}^{(s-2+k-j)} \partial X_{m_{1}} \ldots \partial X_{m_{s-1}} \\
& \left.\times \psi^{\alpha_{0}} \partial \psi_{\alpha_{1}} \ldots \partial^{k-1} \psi_{\alpha_{k-1}}\left(\partial^{1+j} X_{\alpha_{k}}\right) \partial^{k+1} \psi_{\alpha_{k+1} \ldots \partial^{s-3}} \psi_{\alpha_{s-3}}\right] \\
& -2(s-1) \sum_{k=0}^{s-1} \frac{1}{k !} B_{\phi-\chi}^{(s-1-k)} \partial X_{m_{1}} \ldots \partial X_{m_{s-2}} \partial^{j} \psi_{m_{s-1}} \psi^{\alpha_{0}} \partial \psi_{\alpha_{1}} \partial^{2} \psi_{\alpha_{2}} \ldots \partial^{s-3} \psi_{\alpha_{s-3}} \\
& A_{2}(p ; u)=-4(2 s-3) \Omega_{\alpha_{0}}^{m_{1} \ldots m_{s-1} \mid \alpha_{1} \ldots \alpha_{s-3}}(p) \\
& \times \oint d z(z-u)^{2 s-4} \partial c c e^{2 \chi+(s-4) \phi} \psi^{\alpha_{0}} \partial \psi_{\alpha_{1}} \partial^{2} \psi_{\alpha_{2}} \ldots \partial^{s-3} \psi_{\alpha_{s-3}} e^{i p X}(u)
\end{aligned}
$$


with $A_{0}, A_{1}$ and $A_{2}$ terms having the ghost structure described above, namely, $e^{(s-2) \phi}, c e^{(s-3) \phi+\chi}$ and $\partial c c e^{(s-4) \phi+2 \chi}$ respectively and the integrals being taken around an arbitrary point $u$ on the worldsheet. The choice of $u$ is arbitrary as any correlators involv$\operatorname{ing} V_{s-1 \mid s-3}^{(+)}(p ; u)$-operators are $u$-independent since their $u$-derivatives are BRST-exact in the small Hilbert space; the operators themselves can be cast as BRST commutators in the large Hilbert space (but not in the small Hilbert space) and therefore are the elements of BRST cohomology in the small Hilbert space ,given the on-shell conditions on $\Omega$ and modulo the gauge transformations [48], [45]. The $B_{m \phi+n \chi+p \sigma}^{(n)}$ are the normalized degree $n$ Bell polynomials in the bosonized superconformal ghost fields $\phi, \chi$ and $\sigma$, defined according to

$$
\partial_{z}^{n} e^{m \phi+n \chi+p \sigma}(z) \equiv \frac{1}{n !}: B_{m \phi+n \chi+p \sigma}^{(n)} e^{m \phi+n \chi+p \sigma}(z)
$$

where $m, n, p$ are some numbers. The properties and the operator algebras involving the Bell polynomial operators have been studied in details in [47] and will be used in the present calculation. Although the negative and positive picture representations of the higher spin vertex operators $V_{s-1 \mid s-3}^{( \pm)}(p ; u)$ are not directly related by picture-changing transformations (as is clear from the fact that they belong to the nonzero negative/positive cohomologies), they can be mapped to each other by combining BRST-invariant picturechanging and $Z$-transformations which ensures that the on-shell BRST-invariance constraints on $\Omega$ are identical in the both of the representations [44], [45]. Technically, it is easier to analyze these constraints at negative pictures, with the BRST invariance imposing the on-shell conditions:

$$
\begin{aligned}
& p^{2} \Omega_{\alpha_{0}}^{m_{1} \ldots m_{s-1} \mid \alpha_{1} \ldots \alpha_{s-3}}(p)=0 \\
& \Omega_{\alpha_{0} m}^{m m_{3} \ldots m_{s-1} \mid \alpha_{1} \ldots \alpha_{s-3}}(p)=0 \\
& \Omega_{\alpha_{0} \alpha}^{m_{1} \ldots m_{s-1} \mid \alpha \alpha_{3} \ldots \alpha_{s-3}}(p)=0 \\
& \Omega_{\alpha_{0} \alpha}^{m_{2} \ldots m_{s-1} \mid \alpha \alpha_{2} \ldots \alpha_{s-3}}(p)=0
\end{aligned}
$$

The first and the second constraints are the standard constraints for the symmetric higherspin fields in the frame-like description. They are supplemented by two more constraints, indicating that the vertex operators (6), (7) describe the frame-like fields in space-time with the gauge partially fixed. The gauge transformations for $\Omega$ :

$$
\Omega_{m}^{a_{1} \ldots a_{s-1} \mid b_{1} \ldots b_{s-3}} \rightarrow \Omega_{m}^{a_{1} \ldots a_{s-1} \mid b_{1} \ldots b_{s-3}}+p_{m} \Lambda^{a_{1} \ldots a_{s-1} \mid b_{1} \ldots b_{s-3}}
$$

in turn shift the operators (6), (7) by the BRST-exact term (see 44, 445 for the detailed BRST analysis). It is essential that the $\beta$-function equations for the $\Omega$-field, in the leading 
order, defining the kinetic term for the corresponding Fronsdal's field and obtained using the off-shell Weyl invariance constraints on the operators (6), (7) differ from the on-shell BRST-invariance constraints due to nontrivial ghost couplings of the higher spin vertex operators. Namely, the leading order $\beta_{\Omega}=0$ equations are equivalent to $\hat{L}_{A d S} \Omega=0$ where $\hat{L}_{A d S}$ is the Fronsdal's kinetic operator in the $A d S$, rather than flat space, as was shown by detailed computations in [45]. In particular, the appearance of the mass-like term in $\hat{L}_{A d S}$ (which is identified with the tail of the covariant derivatives in the AdS Laplacian) is directly related to the Weyl transformations of the anomalous superconformal ghost parts of the higher spin vertex operators.

For the lower spin vertex operators, such as a graviton, there is a familiar example, somewhat reminiscent of this difference between Weyl and BRST transformations: e.g. recall that the graviton's $\beta$-function contains a term given by the second derivative of the dilaton (which is of course absent in the on-shell conditions imposed by the BRSTinvariance). For this reason, it is natural to think of the $(m, \alpha)$ indices in (11) as of those living in the tangent bundle of emergent $A d S$ space. Accordingly, the correlators of these operators describe the $A d S$ higher spin interactions, upon the pullback from the bundle to the manifold.

For the $t$ values other than $t=s-3$ the manifest expressions for the higher spin vertex operators become significantly more complicated.

The explicit relation between the vertex operators $V_{s-1 \mid t} \equiv \Omega^{s-1 \mid t} W_{s-1 \mid t}$ (with $W$ being conformal dimension 0 primary fields which, upon coupling with the space-time higher spin frame-like fields $\Omega^{s-1 \mid t}$ are the elements of $H_{-s}$ ) with different $t$ values , generating the chain of the zero torsion constraints, is given by (modulo the on-shell constraints (11))

$$
\Omega^{s-1 \mid t}: \Gamma W_{s-1 \mid t}:=\Omega^{s-1 \mid t+1} W_{s-1 \mid t+1}
$$

Here $W$ are the conformal dimension 0 vertex operators which, upon coupling to the space-time higher spin frame-like fields $\Omega^{s-1 \mid t}$, become the elements of $H_{-s} . \Gamma$ is the picture-changing operator for the $\beta-\gamma$ ghost system given by

$$
\Gamma=\left\{Q, e^{\chi}\right\}=-\frac{1}{2} e^{\phi} \psi_{m} \partial X^{m}+\frac{1}{4} b e^{2 \phi-\chi}\left(\partial_{\chi}+\partial \sigma\right)+c e^{\chi} \partial \chi
$$

The relation (13) can be generalized according to

$$
\begin{array}{r}
\Omega^{s-1 \mid t}: \Gamma^{k} W_{s-1 \mid t}:=\Omega^{s-1 \mid t+1} W_{s-1 \mid t+k}(k \leq s-3-t) \\
\Omega^{s-1 \mid t}: \Gamma^{k} W_{s-1 \mid t}:=0(k>s-3-t)
\end{array}
$$


where $\Gamma^{k}=: \Gamma \ldots \Gamma:=: e^{k \phi} G \partial G \ldots \partial^{k-1} G$ : is the normally ordered product of $k$ picturechanging operators and $G$ is the full matter+ghost worldsheet supercurrent. In particular, to obtain the operator for the Fronsdal's field one can take $k=s-3$ and $t=0$.

$k=s-3$ Note that all the $V_{s-1 \mid t} \equiv \Omega^{s-1 \mid t} W_{s-1 \mid t}$ higher spin vertex operators are the elements of $H_{-s} \sim H_{s-2}$ cohomology for all the values of $0 \leq t \leq s-3$, although the canonical pictures for $V_{s-1 \mid t}$ are different and equal to $-2 s+t+3$ at the negative picture representation. The action of $\Gamma$ on $V_{s \mid t}$ thus results in increasing the ghost picture by one unit and the appearance of the extra $p$ factor in front of $\Omega(p)$, typically due to the contraction of the first term in $\Gamma$ with $e^{i p X}$ factor in the vertex operator. The zero torsion constraints can of course be reformulated equivalently for the operators in the positive cohomology representations; will all the spin $s$ operators being the elements of $H_{s-2}$, the constraints for the frame-like vertex operators in the positive cohomologies are

$$
\begin{array}{r}
\Omega^{s-1 \mid t}: \Gamma^{-k} W_{s-1 \mid t}:=\Omega^{s-1 \mid t+1} W_{s-1 \mid t+k}(k \leq s-3-t) \\
\Omega^{s-1 \mid t}: \Gamma^{-k} W_{s-1 \mid t}:=0(k>s-3-t)
\end{array}
$$

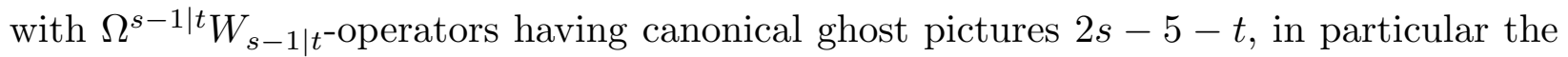
operator for the Fronsdal's field having canonical positive picture $2 s-5$. Here $: \Gamma^{-k}:=$ : $\left(\Gamma^{-1}\right)^{k}$ : with $\Gamma^{-1}=-4 c e^{\chi-2 \phi} \partial \chi$ being the inverse picture-changing operator.

In practice, the equations (15), (16) generating the generalized zero torsion constraints by the picture-changing relations, are hard to solve. For example, the solution for the simplest of the equations (15) for $t=s-4$ is already complicated enough, with the vertex operator having the form

$$
\begin{array}{r}
V_{s-1 \mid s-4}(p ; z) \sim c e^{-(s+1) \phi} \partial X_{m_{1}} \ldots \partial X_{s-1} \psi^{\alpha_{0}} \partial \psi_{\alpha_{1}} \partial^{2} \psi_{\alpha_{2}} \ldots \partial^{s-4} \psi_{\alpha_{s-4}} \\
\times\left[\alpha B_{-\phi}^{(2 s-3)} \sum_{p=0}^{s-3} \sum_{q=p+1}^{s-2} \alpha_{p \mid q} \partial^{p} \psi_{m} \partial^{q} \psi^{n} B_{-\phi}^{(2 s-4-p-q)}+\alpha_{s-3 \mid s-1} \partial^{s-3} \psi_{m} \partial^{s-1} \psi^{m}\right] e^{i p X}
\end{array}
$$

at canonical $-s-1$-picture where the $\alpha_{p \mid q}$-coefficients must be calculated so as to ensure that $V_{s-1 \mid s-4}(p ; z)$ is primary (i.e. the singularities of cubic and higher orders stemming from the OPE of the stress-tensor with the $\psi$-part must be cancelled by those stemming from the operator product with the Bell polynomials in the derivatives of the $\phi$-ghost field). Note that the leading order of the operator products of all the Bell polynomials $B_{-\phi}^{(N)}$ with $e^{\phi}$ is the simple pole for any $N$, which ensures that the picture-changing transformation of (17) does not produce any terms other than those proportional to $V_{s-1 \mid s-3}$, as well as 
the absence of singularities in the OPE of $\Gamma$ and $V_{s-1 \mid s-4}(p ; z)$. It is now not hard to see that the expressions for operators with $t \leq s-5$ will get more and more tedious for the lower $t$ values; their general structure would involve sums over multiple products of $\partial^{p} \vec{\psi} \partial^{q} \vec{\psi}$-factors multiplied by products of $s-3-t$ Bell polynomials of the ghost fields, making them cumbersome objects to work with.

Thus the $V_{s-1 \mid s-3}$-operators appear to be particularly convenient and natural objects to use in order to describe the higher-spin vertices in space-time. However, there are restrictions on the spin values for the vertices that can be described by the correlators with all the operators being of the $V_{s-1 \mid s-3}$-only. That is, for a $n=p+q$-point higher spin amplitude containing $p V_{s-1 \mid s-3}$-operators at positive cohomologies and $q$ operators at negative cohomologies the spin values must satisfy

$$
\sum_{j=1}^{p}\left(s_{j}-2\right)-\sum_{j=p+1}^{p+q} s_{j}=-2
$$

in order to satisfy the superconformal ghost balance constraint. The amplitudes with spin values not satisfying (18) cannot be described by using solely the operators of this type and require finding explicit solutions of the operator equations (15), (16) making them far more complicated. At the same time, note that if the constraint (18) is satisfied, despite the fact that the correlation functions of the $V_{s-1 \mid s-3}$-type operators by construction contain certain minimal number of derivatives (since each space-time field $\Omega_{s-1 \mid s-3}$ by definition contains $s-3$ derivatives), all the amplitudes involving $V_{s-1 \mid s-3}$ can be cast equivalently in terms of those involving operators for the Fronsdal's fields and/or the operators for the extra fields $\Omega_{s-1 \mid t}$ with lower $t$, using the zero torsion relations (15), (16), combined with the picture equivalence of the operators inside each particular cohomology. For example, consider a 5-point higher spin amplitude with the spins satisfying the constraints (18): $s_{1}+s_{2}-s_{3}-s_{4}-s_{5}=2$. In the amplitude of this type, two operators are integrated at positive picture, and three operators are unintegrated at negative pictures. Using the zero torsion relations (15), (16) we get

$$
\begin{array}{r}
A\left(s_{1}, \ldots s_{5}\right)=<\Omega^{s_{1}-1 \mid s_{1}-3} W_{s_{1}-1 \mid s_{1}-3}^{\left(s_{1}-2\right)}\left(p_{1}\right) \Omega^{s_{2}-1 \mid s_{2}-3} W_{s_{2}-1 s_{2}-3}^{\left(s_{2}-2\right)}\left(p_{2}\right) \\
\Omega^{s_{3}-1 \mid s_{3}-3} W_{s_{3}-1 \mid s_{3}-3}^{\left(-s_{3}\right)}\left(p_{3}\right) \Omega^{s_{4}-1 \mid s_{4}-3} W_{s_{4}-1 \mid s_{4}-3}^{\left(-s_{4}\right)}\left(p_{4}\right) \Omega^{s_{5}-1 \mid s_{5}-3} W_{s_{5}-1 \mid s_{5}-3}^{\left(-s_{5}\right)}\left(p_{5}\right)> \\
=<: \Gamma^{-s_{1}-s_{2}+6}: \Omega^{s_{1}-1 \mid 0} W_{s_{1}-1 \mid s_{1}-3}^{\left(2 s_{1}-5\right)}\left(p_{1}\right) \Omega^{s_{2}-1 \mid 0} W_{s_{2}-1 s_{2}-3}^{\left(2 s_{2}-5\right)}\left(p_{2}\right) \\
: \Gamma^{s_{3}+s_{4}+s_{5}-9}: \Omega^{s_{3}-1 \mid 0} W_{s_{3}-1 \mid 0}^{\left(3-2 s_{3}\right)}\left(p_{3}\right) \Omega^{s_{4}-1 \mid 0} W_{s_{4}-1 \mid 0}^{\left(3-2 s_{4}\right)}\left(p_{4}\right) \Omega^{s_{5}-1 \mid 0} W_{s_{5}-1 \mid 0}^{\left(3-2 s_{5}\right)}\left(p_{5}\right)> \\
=<\Omega^{s_{1}-1 \mid 0} W_{s_{1}-1 \mid 0}^{\left(2 s_{1}-6\right)}\left(p_{1}\right) \Omega^{s_{2}-1 \mid 0} W_{s_{2}-1 \mid 0}^{\left(2 s_{2}-6\right)}\left(p_{2}\right) \Omega^{s_{3}-1 \mid 0} W_{s_{3}-1 \mid 0}^{\left(2-2 s_{3}\right)}\left(p_{3}\right) \\
\Omega^{s_{4}-1 \mid 0} W_{s_{4}-1 \mid 0}^{\left(2-2 s_{4}\right)}\left(p_{4}\right) \Omega^{s_{5}-1 \mid 0} W_{s_{5}-1 \mid 0}^{\left(2-2 s_{5}\right)}\left(p_{5}\right)>
\end{array}
$$


i.e. the amplitude involving five $V_{s-1 \mid s-3}$ higher spin operators is identical to the one involving five Fronsdal operators at pictures each lowered by one unit with respect to the canonical.

As it is clear from the above discussion, the overall $b-c$ and superconformal ghost structures of the $n=p+q$-point higher spin amplitudes, combined with the ghost structure of the picture-changing operators (14) suggest the existence of two types of higher spin interactions at higher orders: the first type having $p=n-3, q=3$. This amplitude involves 3 unintegrated negative picture operators and standard $N-3$ integrated operators at positive pictures. All the terms, contributed by all the integrated operators, are of $A_{0}$-type (having $b-c$ ghost number zero) The higher spin amplitudes of this type have the standard Veneziano pole structure, leading to local interaction terms in the low-energy effective action (with the poles in the amplitudes corresponding to different channels of particle exchanges). These poles do not produce any physical nonlocalities (the nonlocalities that one may encounter upon the space-time momentum integration and expressing the lowenergy effective action in the position space, are not physical and can be removed by substituting the $\beta$-function equations at lower orders [46] (strictly speaking, the locality of the amplitude does not by itself guarantee the locality of the related higher spin interaction vertex in the low-energy effective action; for the case, considered in this paper see, however, the discussion below in the section 4)

The amplitudes of the second type, on the other hand, have the structure $p=n-$ $2, q=2$.They involve two unintegrated operators contributing two $c$-ghosts, with the third $c$-ghost stemming from the $A_{1}$-type terms of one of the integrated operators and the remaining operators contributing $A_{0}$-type terms. These amplitudes have the structure very different from those of the first type. Due to the extra integration, they contain extra poles, corresponding to physical nonlocalities, rather than particle exchanges. Unlike the Veneziano-type case, the nonlocalities in the position space, obtained upon the Fourier transform, cannot be removed using the $\beta$-function flows, but reflect genuine nonlocalities of the higher spin interactions.

In fact, generically most of the higher spin amplitudes are of the second type, with the first type emerging only for the special combination of the spin values. We will refer to the appearance of the first type higher spin amplitudes as the "localization". In fact, as we shall point out below, such a localization effect does not occur at the quartic order but only appears at quintic and higher order interactions. In the next section, we will study this effect by direct computation of the correlation functions. 


\section{Quintic Interactions and Localization}

We start with the 4-point higher spin amplitude with the spin values satisfying constraint (18) with $p=1, q=3$. It is not difficult to see that this 4-point amplitude vanishes. Indeed, the $V_{s_{1}}$ operator at positive cohomology contains $s_{1}-2=s_{1}+s_{2}+s_{3}-2 \psi$-fields, which cannot fully contract to the RNS fermions of the remaining 3 operators, since the operators at negative pictures contribute altogether $s_{1}+s_{2}+s_{3}-6 \psi$-fields. This means that the 4-point amplitude with such spin values admits no localization. Next, consider the 5 -point amplitude with the localization constraint $p=2, q=3$. To simplify the calculations as much as possible, we assume that the value one of the spins is small enough (namely, $s_{4} \equiv u=4$ ) and $s_{5}+u-s_{1}-s_{2}-s_{3}=2$. All the vertex operators for the frame-like fields are in the $V_{s-1 \mid s-3}$ representation, with the operators the $s_{1}, s_{2}, s_{3}$ being unintegrated at negative pictures and the operators for spins $u=4$ and $s_{5}=\sum s-2$ at integrated positive (to abbreviate the notations, denote $\sum s=s_{1}+s_{2}+s_{3}$ ). With such a picture arrangement, only $A_{0}$-type terms of the both of the integrated operators contribute to the correlator. We are now all set to compute, step by step, the $X$, ghost and $\psi$-factors of the correlator defining the quintic interaction of the above spin values. We start with the calculation of the $X$-part. The correlation function is given by

$$
\begin{gathered}
A_{X}\left(p_{1}, \ldots p_{5} \mid w ; z ; \xi\right)=<\left.\partial X^{m_{1}} \ldots \partial X^{m_{s_{1}-1}} e^{i p_{5} X}(w)\right|_{w \rightarrow \infty} \partial X^{n_{1}} \ldots \partial X^{n_{s_{2}-1}} e^{i p_{4} X}(1) \\
\partial X^{q_{1}} \ldots \partial X^{q_{u-1}} e^{i p_{3} X}(z) \partial X^{r_{1}} \ldots \partial X^{{ }^{r} \sum^{s+1-u}} e^{i p_{2} X}(\xi) \partial X^{t_{1}} \ldots \partial X^{t_{s_{3}-1}} e^{i p_{1} X}(0)>
\end{gathered}
$$

where we have arranged the operator's insertions in the order $\left(z_{1}=w\right)>\left(z_{2}=1\right)>$ $z>\xi>\left(z_{3}=0\right)$, with $z_{1,2,3}$ being the locations of the spin $s_{1,2,3}$ unintegrated vertices, $z$ and $\xi$ are the insertion points of the remaining spins (to be integrated over). We shall later set $z_{3} \equiv w \rightarrow \infty$ but for now shall the $w$-dependence manifest to keep track of the infinities. To compute the correlator (20), define the partitions

$$
s_{i}-1=\sum_{j=1, j \neq i}^{5}\left(R_{i j}+Q_{i j}\right) ; i, j=1, \ldots, 5
$$

where $R_{i j}$ is the number of contractions between $\partial X$ 's of the operators of spins $s_{i}$ and $s_{j}$ (obviously $R_{i j}=R_{j i}$ ) and $Q_{i j}$ counts the contractions of $X$-derivatives of the operators for $s_{i}$ with the exponent $e^{i p X}$ in the operator for spin $s_{j}$. Straightforward computation 
then gives:

$$
\begin{aligned}
& A_{X}\left(p_{1}, \ldots p_{5} \mid w ; z ; \xi\right) \\
& =\sum_{\left[\text {partitions } s_{i}-1 \mid \sum_{j=1, j \neq i}^{5}\left(R_{i j}+Q_{i j}\right) ; i=1, \ldots, 5\right]} \frac{6\left(s_{1}-1\right) !\left(s_{2}-1\right) !\left(s_{3}-1\right) !\left(\sum s-3\right) !}{\prod_{i=1}^{4} \prod_{j=2 ; j>i}^{5} R_{i j} ! \prod_{k=1}^{5} \prod_{l=1 ; k \neq l}^{5} Q_{k l} !} \\
& (i)^{\sum_{i, j=1 ; i \neq j}^{5} Q_{i j}}(-1)^{\sum_{j=1}^{5}\left(Q_{1 j}+Q_{2 j}\right)-Q_{21}+Q_{43}+Q_{43}+Q_{45}+Q_{53}} w^{-\sum_{j=2}^{5}\left(2 R_{1 j}+Q_{1 j}+Q_{1 j}\right)} \\
& \times(1-z)^{p_{3} p_{4}-2 R_{24}-Q_{24}-Q_{42}}(1-\xi)^{p_{2} p_{4}-2 R_{25}-Q_{25}-Q_{52}} \\
& (z-\xi)^{p_{2} p_{3}-2 R_{45}-Q_{45}-Q_{54}} z^{p_{1} p_{3}-2 R_{34}-Q_{34}-Q_{43}} \xi^{p_{1} p_{2}-2 R_{35}-Q_{35}-Q_{53}} \\
& \times p_{1}^{m_{Q_{12}+Q_{14}+Q_{15}+1}} \ldots p_{1}^{m_{Q_{12}}+Q_{14}+Q_{15}+Q_{13}} p_{1}^{n_{Q_{21}+Q_{24}+Q_{25}+1}} \ldots p_{1}^{n_{Q_{21}+Q_{24}+Q_{25}+Q_{23}}} \\
& p_{1}^{q_{Q_{41}+Q_{42}+Q_{45}+1}} \ldots p_{1}^{q_{Q_{41}+Q_{42}+Q_{45}+Q_{43}}} p_{1}^{r_{Q_{51}+Q_{52}+Q_{54}+1}} \ldots p_{1}^{r_{Q_{51}+Q_{52}+Q_{54}+Q_{53}}}
\end{aligned}
$$

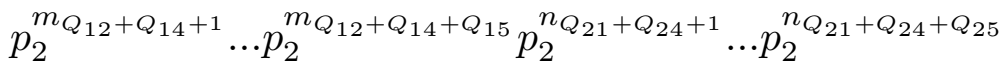

$$
\begin{aligned}
& p_{2}^{q_{Q_{41}+Q_{42}+1}} \ldots p_{2}^{q_{Q_{41}+Q_{42}+Q_{45}}} p_{2}^{t_{Q_{31}+Q_{32}+Q_{34}+1}} \ldots p_{2}^{t_{Q_{31}+Q_{32}+Q_{34}+Q_{35}}} \\
& \times p_{3}^{m_{Q_{12}+1}} \ldots p_{3}^{m_{Q_{12}+Q_{14}}} p_{3}^{n_{Q_{21}+1}} \ldots p_{3}^{n_{Q_{21}+Q_{24}}} \\
& p_{3}^{r_{Q_{51}+Q_{52}+1}} \ldots p_{3}^{r_{Q_{51}+Q_{52}+Q_{54}}} p_{3}^{t_{Q_{31}+Q_{32}+1}} \ldots p_{3}^{t_{Q_{31}+Q_{32}+Q_{34}}} \\
& p_{4}^{m_{1}} \ldots p_{4}^{m_{Q_{12}}} p_{4}^{q_{Q_{41}+1}} \ldots p_{4}^{q_{Q_{41}+Q_{42}}} p_{4}^{r_{Q_{51}+1}} \ldots p_{4}^{r_{Q_{51}+Q_{52}}} p_{4}^{t_{Q_{31}+1}} \ldots p_{4}^{t_{Q_{31}+Q_{32}}} \\
& p_{5}^{n_{1}} \ldots p_{5}^{n_{Q_{21}}} p_{5}^{q_{1}} \ldots p_{5}^{q_{Q_{41}}} p_{5}^{r_{1}} \ldots p_{5}^{r_{Q_{51}}} p_{5}^{t_{1}} \ldots p_{5}^{t_{Q_{31}}} \\
& \times \eta^{m} \sum_{j} Q_{1 j}{ }^{\mid n} \sum_{j}{ }^{Q_{2 j}+1} \ldots \eta^{m} \sum_{j}{ }^{Q_{1 j}+R_{12}}{ }^{\mid n} \sum_{j}{ }^{Q_{2 j}+R_{12}} \\
& \eta^{m} \sum_{j} Q_{1 j}+1+R_{12}{ }^{\mid q} \sum_{j}{ }^{Q_{4 j}+1} \ldots \eta^{m} \sum_{j} Q_{1 j}+R_{12}+R_{14}{ }^{\mid q} \sum_{j} Q_{4 j}+R_{14}
\end{aligned}
$$

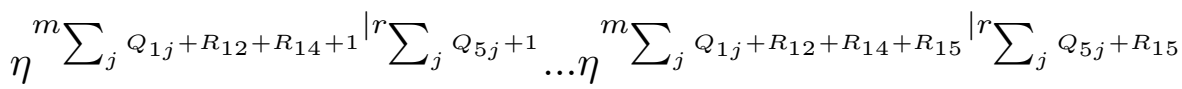

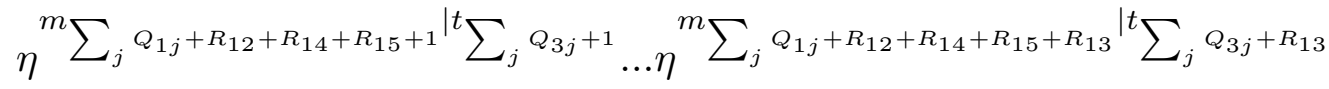

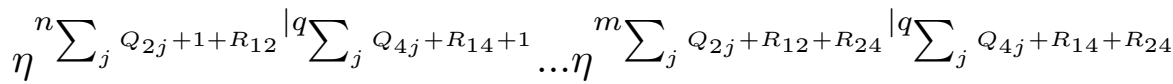

$$
\begin{aligned}
& \eta^{n} \sum_{j} Q_{2 j}+1+R_{12}+R_{24}{ }^{\mid r} \sum_{j} Q_{5 j}+R_{15}+1 \ldots \eta^{m} \sum_{j} Q_{2 j}+R_{12}+R_{24}+R_{25}{ }^{\mid r} \sum_{j} Q_{5 j}+R_{15}+R_{25}
\end{aligned}
$$

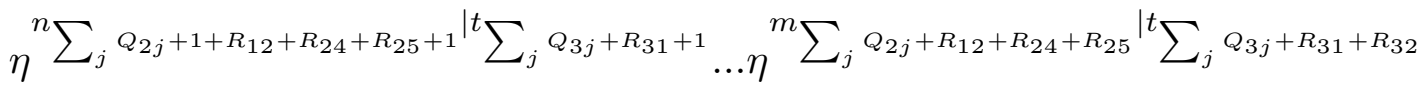

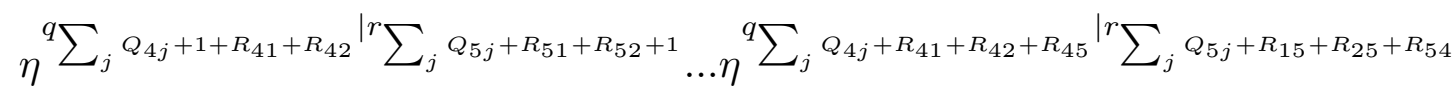

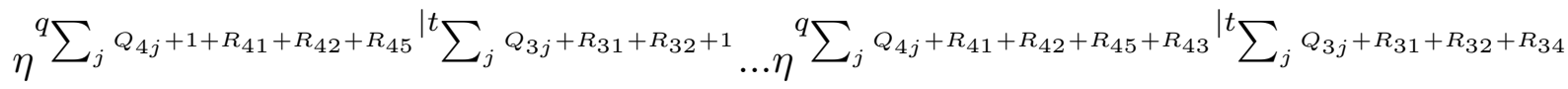

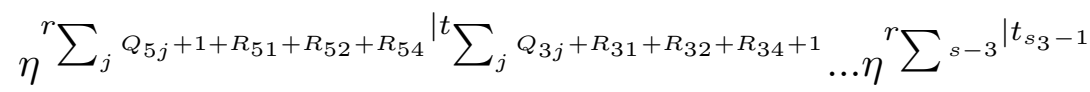


where in our notations $\eta^{m_{i} \mid n_{j}}$ is Minkowski tensor and $\sum_{j} A_{i j} \equiv \sum_{j=1 ; j \neq i}^{5} A_{i j}$

This concludes the $X$-part evaluation. Next, consider the ghost part of the amplitude. The relevant correlator is

$$
A_{g h}(w ; z ; \xi)=<c e^{-s_{1} \phi}(w) c e^{-s_{2} \phi}(1) e^{(u-2) \phi} B_{2 \phi-2 \chi-\sigma}^{(2 u-2)}(z) e^{(\Sigma s-u) \phi} B_{2 \phi-2 \chi-\sigma}^{(2 \Sigma s-u)}(\xi) c e^{-s_{3} \phi}(0)>
$$

To calculate it, introduce again the characteristic partitions:

$$
\begin{array}{r}
2(u-2)=4=\alpha_{1}+\alpha_{2}+\alpha_{3}+\alpha_{4}^{(1)}+\alpha_{4}^{(2)} \\
2(\Sigma s-u) \equiv 2(\Sigma s-4)=\beta_{1}+\beta_{2}+\beta_{3}+\beta_{4}^{(1)}+\beta_{4}^{(2)}
\end{array}
$$

where the integers $\alpha_{j}(j=1,2,3)$ refer to the OPE singularity orders $\sim\left(z-z_{j}\right)^{-\alpha_{j}}$ due to contractions of $B_{2 \phi-2 \chi-\sigma}^{(2 u-2)}(z)$ with the ghost exponents $c e^{-s_{j} \phi}\left(z_{j}\right) ; \beta_{j}(j=1,2,3)$ refer to the OPE singularities $\sim\left(\xi-z_{j}\right)^{-\beta_{j}}$ due to contractions of $B_{2 \phi-2 \chi-\sigma}^{(2 \Sigma s-u)}(\xi)$ with $c e^{-s_{j} \phi}\left(z_{j}\right)$; $\alpha_{4}^{(1)} \beta_{4}^{(1)}$ refer to the OPE singularities of the Bell polynomials at $z$ and $\xi$ with the opposite ghost exponents $e^{(\Sigma s-u) \phi}(\xi)$ and $B_{2 \phi-2 \chi-\sigma}^{(2 u-2)}(z)$ respectively and, finally $\alpha_{4}^{(2)}$ and $\beta_{4}^{(2)}$ refer to the OPE due to contractions between Bell polynomials at $z$ and $\xi$, i.e.

$$
\sim \sum_{\alpha_{4}^{(2)}, \beta_{4}^{(2)}}(z-\xi)^{-\alpha_{4}^{(2)}-\beta_{4}^{(2)}} \lambda\left(\alpha_{4}^{(2)}, \beta_{4}^{(2)-2)}\right): B_{2 \phi-2 \chi-\sigma}^{\left(2 u-2-\alpha_{4}^{(2)}\right)}(z) B_{2 \phi-2 \chi-\sigma}^{\left(2 \Sigma s-u-\beta_{4}^{(2)}\right)}(\xi):
$$

where $\lambda\left(\alpha_{4}^{(2)}, \beta_{4}^{(2)}\right)$ are the structure constants that can be computed (see below). The correlator (23) is thus equal to the universal factor due to contractions between the ghost exponents, multiplied by the sum stemming from contractions of the Bell polynomials with themselves and with various exponents, with each term in the sum corresponding to some of the partitions (24), with the correlator being given by the sum over the partitions. The explicit expressions for the operator products, needed to compute the correlator, are 47

$$
B_{\vec{\alpha} \vec{\varphi}}^{\left(N_{1}\right)}(z) B_{\vec{\beta} \vec{\varphi}}^{\left(N_{2}\right)}(w)=\sum_{n_{1}=0}^{N_{1}} \sum_{n_{2}=0}^{N_{2}}(z-w)^{-n_{1}-n_{2}} \lambda\left(n_{1}, n_{2}\right): B_{\vec{\alpha} \vec{\varphi}}^{\left(N_{1}\right)}(z) B_{\vec{\beta} \vec{\varphi}}^{\left(N_{2}\right)}(w):
$$

and

$$
B_{\vec{\alpha} \vec{\varphi}}^{(N)}(z) e^{\vec{\beta} \vec{\varphi}}(w)=\sum_{n=0}^{N}(z-w)^{-n} \frac{\Gamma(-\vec{\alpha} \vec{\beta}+1)}{n ! \Gamma(-\vec{\alpha} \vec{\beta}+1-n)}: B_{\vec{\alpha} \vec{\varphi}}^{(N-n)}(z) e^{\vec{\beta} \vec{\varphi}}(w):
$$

where

$$
\begin{array}{r}
\vec{\alpha} \vec{\varphi} \equiv \alpha_{1} \phi+\alpha_{2} \chi+\alpha_{3} \sigma \\
\vec{\alpha} \vec{\beta}=\alpha_{1} \beta_{1}-\alpha_{2} \beta_{2}-\alpha_{3} \beta_{3}
\end{array}
$$


and the OPE structure constants in (26) are given by 47

$$
\begin{array}{r}
\lambda\left(n_{1}, n_{2}\right)=\left.\frac{1}{n_{1} ! n_{2} !} \partial_{x}^{n_{1}} \partial_{y}^{n_{2}} F_{\lambda}(x, y)\right|_{x=y=0} \\
F_{\lambda}(x, y)=\left[\frac{(1+x)(1-y)}{1+x-y}\right]^{\vec{\alpha} \vec{\beta}}
\end{array}
$$

Clearly, in our case $\vec{\alpha} \vec{\beta}=-1$ so one simply has

$$
\lambda\left(n_{1}, n_{2}\right)=(-1)^{n_{1}}
$$

With all the above identities it is now straightforward to compute the ghost correlator, with the result given by:

$$
\begin{array}{r}
=\sum_{\left[\text {partitions: } 2(u-2) \equiv 4 \mid \alpha_{1}+\alpha_{2}+\alpha_{3}+\alpha_{4}^{(1)}+\alpha_{4}^{(2)}\right]} A_{g h}(w ; z ; \xi) \\
\sum_{\left[\text {partitions }: 2(\Sigma s-u) \equiv 2(\Sigma s-4) \mid \beta_{1}+\beta_{2}+\beta_{3}+\beta_{4}^{(1)}+\beta_{4}^{(2)}\right]} \\
\prod_{j=1}^{3} \frac{\left(\left(2 s_{j}-1\right) !\right)^{2}}{\Gamma(3-2 u) \Gamma(1+2 u-2 \Sigma s)} \\
\times(-1)^{\alpha_{4}^{(2)}+1} w^{s_{1}^{2}+2-\alpha_{1}-\beta_{1}}(1-z)^{2 s_{2}-\alpha_{2}}(1-\xi)^{s_{2}(\Sigma s-4)-\beta_{2}} \\
\times z^{2 s_{3}-\alpha_{3}} \xi^{s_{3}(\Sigma s-4)-\beta_{3}}(z-\xi)^{2(4-\Sigma s)-\alpha_{4}^{(1)}-\alpha_{4}^{(2)}-\beta_{4}^{(1)}-\beta_{4}^{(2)}}
\end{array}
$$

This concludes the computation of the ghost factor, contributing to the integrand of the 5-point amplitude. Finally, we are left with computing the $\psi$-factor, given by

$$
<\prod_{j_{1}=0}^{s_{1}-3} \partial^{j_{1}} \psi_{\alpha_{j_{1}}}(w) \prod_{j_{2}=0}^{s_{2}-3} \partial^{j_{2}} \psi_{\beta_{j_{2}}}(1) \prod_{j_{3}=0}^{u-3} \partial^{j_{3}} \psi_{\gamma_{j_{3}}}(z) \prod_{j_{4}=0}^{\Sigma s-u-1} \partial^{j_{4}} \psi_{\lambda_{j_{4}}}(\xi) \prod_{j_{5}=0}^{s_{3}-3} \partial^{j_{5}} \psi_{\sigma_{j_{5}}}(0)>
$$

This amplitude would be actually the most tedious one to compute for generic spin values, since there seems to be no way to systemize it in terms of sums over partitions of the spin values, as it has been done for the previous correlators. However, assuming that $u$ is the minimal spin value of the operators, it simplifies significantly for the minimal possible 
$u=4$ value (for $u<4$ it vanishes identically). The simplification is that for $u=4$, all the $\psi$-fields of 4 operators have to couple to the $\psi$-fields of the operator with the highest spin value, given by $s_{\max }=\Sigma s-2$. Technically, this means that in the corresponding spacetime amplitude all the 4 out of $5 \Omega^{s-1 \mid t}$ extra-field's $t$-indices $\alpha, \beta, \gamma, \sigma$ have to contract to the $\lambda$-index of the highest spin field $\Omega^{s_{\max }-1 \mid \lambda}$. The computation performed below is still possible to perform for $u>4$, e.g. for $u=5$ or 6 , however, for $u>4$ the locality of the interaction vertex in space-time would be broken by the RG flows from lower orders despite the locality of the scattering amplitude (see the discussion in the Section 4) In case of $u=4$, it is convenient to make the following definition. Let $i\left(\alpha_{k}\right)$ describe the contraction of the worldsheet fermion $\partial^{k} \psi_{\alpha_{k}}$ of the spin $s_{1}$ operator to the fermion $\partial^{i\left(\alpha_{k}\right)} \psi_{\lambda_{i\left(\alpha_{k}\right)}}$ of the spin $s_{\max }$ operator. Similarly, let $i\left(\beta_{k}\right)$ describe the contractions between the worldsheet fermions of the $s_{2}$ and $s_{\max }$ operators, and so on. Clearly, $i(\alpha) \neq i(\beta) \neq i(\gamma) \neq i(\sigma)$ for all values of $\alpha, \beta, \gamma, \sigma$ and $0 \leq i(\alpha, \beta, \gamma, \sigma) \leq s_{\max }-3$ Then it is natural to express the $\psi$-correlator in terms of the sum over the permutations of different $i$ 's, or, equivalently, in terms of sum over all possible length $s_{\max }-2$ orderings of unequal integer numbers from 0 to $s_{\max }-3$.

The $A_{\psi}$ correlator is then straightforward to compute in terms of such a sum, with the result given by

$$
\begin{aligned}
& A_{\psi}(w, z, \xi) \\
& =(-1)^{s_{1} s_{2}+s_{1}+s_{2}+s_{3}} \sum_{\left[\text {permutations }:\left\{, i\left(\alpha_{0}\right) \ldots i\left(\alpha_{s_{1}-3}\right), i\left(\beta_{0}\right) \ldots i\left(\beta_{s_{2}-3}\right), i\left(\gamma_{0}\right), i\left(\gamma_{1}\right), i\left(\sigma_{0}\right) \ldots i\left(\sigma_{s_{3}-3}\right)\right\}\right]} \\
& \left\{(-1)^{\pi\left(\left\{, i\left(\alpha_{0}\right) \ldots i\left(\alpha_{s_{1}-3}\right), i\left(\beta_{0}\right) \ldots i\left(\beta_{s_{2}-3}\right), i\left(\gamma_{0}\right), i\left(\gamma_{1}\right), i\left(\sigma_{0}\right) \ldots i\left(\sigma_{s_{3}-3}\right)\right\}\right)}\right. \\
& \times\left(1+i\left(\gamma_{0}\right)\right) !\left(2+i\left(\gamma_{1}\right)\right) ! \\
& \prod_{k_{1}=1}^{s_{1}-2}\left(k_{1}+i\left(\alpha_{k_{1}}-1\right)\right) ! \prod_{k_{2}=1}^{s_{2}-2}\left(k_{2}+i\left(\beta_{k_{2}}-1\right)\right) ! \\
& \times \prod_{k_{3}=1}^{s_{3}-2}\left(k_{3}+i\left(\beta_{k_{3}}-1\right)\right) ! w^{\frac{1}{2}\left(s_{1}-2\right)^{2}+\sum_{l_{1}=0}^{s_{1}-3} i\left(\alpha_{l_{1}}\right)} \\
& \left.(1-\xi)^{\frac{1}{2}\left(s_{2}-2\right)^{2}+\sum_{l_{2}=0}^{s_{2}-3} i\left(\beta_{l_{2}}\right)} \xi^{\frac{1}{2}\left(s_{3}-2\right)^{2}+\sum_{l_{3}=0}^{s_{2}-3} i\left(\sigma_{l_{3}}\right)}(z-\xi)^{2+i\left(\gamma_{0}\right)+i\left(\gamma_{1}\right)}\right\}
\end{aligned}
$$

where

$$
\pi\left(\left\{i\left(\alpha_{0}\right) \ldots i\left(\alpha_{s_{1}-3}\right), i\left(\beta_{0}\right) \ldots i\left(\beta_{s_{2}-3}\right), i\left(\gamma_{0}\right), i\left(\gamma_{1}\right), i\left(\sigma_{0}\right) \ldots i\left(\sigma_{s_{3}-3}\right)\right\}\right)
$$


is the minimal number of the permutations it takes to convert the ordering

$$
\left\{i\left(\alpha_{0}\right) \ldots i\left(\alpha_{s_{1}-3}\right), i\left(\beta_{0}\right) \ldots i\left(\beta_{s_{2}-3}\right), i\left(\gamma_{0}\right), i\left(\gamma_{1}\right), i\left(\sigma_{0}\right) \ldots i\left(\sigma_{s_{3}-3}\right)\right\}
$$

in the argument of $\pi$ into the reference ordering

$$
\left\{0,1,2, \ldots ., s_{\max }-3\right\}
$$

of $s_{\max }-2$ integers. This concludes the computation of the $\psi$-factor contribuiting to the integrand of the amplitude. The final remaining step to determine the amplitude describing the 5-point higher spin interaction is to perform the double worldsheet integration over the positions of the integrated vertices, given by

$$
\begin{array}{r}
A\left(p_{1}, \ldots, p_{5}\right)= \\
\int_{0}^{1} d z \int_{0}^{z} d \xi z^{4} \xi^{2(\Sigma s-4)} A_{\psi}(w ; z ; \xi) A_{g h}(w ; z ; \xi) A_{X}\left(p_{1}, \ldots p_{5} \mid w ; z ; \xi\right)
\end{array}
$$

to set the limit $w \rightarrow \infty$ and to contract the result with the space-time frame-like higher spin fields (in the integral (9) it is convenient to choose the reference $u$-points $u=0$ in the the both of the homotopy transformations for the integrated vertex operators ). Combining $A_{X}, A_{\psi}$ and $A_{g h}$ factors together, evaluating the integral (34) and contracting with the 
frame-like space-time fields, we obtain the overall quintic amplitude, given by

$$
\begin{aligned}
& A\left(p_{1}, \ldots, p_{5}\right)=6 \pi \Omega_{m_{1} \ldots m_{s_{1}-1} \mid \lambda_{0} \ldots \lambda_{s_{1}-3}}\left(p_{5}\right) \Omega_{n_{1} \ldots n_{s_{2}-1} \mid \lambda_{s_{1}-2} \ldots \lambda_{s_{1}+s_{2}-5}}\left(p_{4}\right) \\
& \Omega_{q_{1} q_{2} q_{3} \mid \lambda_{s_{1}+s_{2}-4} \lambda_{s_{1}+s_{2}-3}}\left(p_{3}\right) \Omega_{r_{1} \ldots r_{\Sigma s-3}}^{\lambda_{0} \ldots \lambda_{\Sigma s-5}}\left(p_{2}\right) \Omega_{t_{1} \ldots t_{s_{3}-1} \mid \lambda_{s_{1}+s_{2}-2 \ldots \lambda_{\Sigma s-5}}}\left(p_{1}\right) \\
& \times \sum_{\text {partitions }: s_{i}-1 \mid} \sum_{j=1, j \neq i}^{5}\left(R_{i j}+Q_{i j}\right) ; i=1, \ldots, 5 \\
& \sum \\
& \text { partitions: } 2(u-2) \equiv 4 \mid \alpha_{1}+\alpha_{2}+\alpha_{3}+\alpha_{4}^{(1)}+\alpha_{4}^{(2)} \\
& \sum_{\text {partitions }: 2(\Sigma s-u) \equiv 2(\Sigma s-4) \mid \beta_{1}+\beta_{2}+\beta_{3}+\beta_{4}^{(1)}+\beta_{4}^{(2)}} \\
& \sum \\
& \text { permutations: }\left\{, i\left(\alpha_{0}\right) \ldots i\left(\alpha_{s_{1}-3}\right), i\left(\beta_{0}\right) \ldots i\left(\beta_{s_{2}-3}\right), i\left(\gamma_{0}\right), i\left(\gamma_{1}\right), i\left(\sigma_{0}\right) \ldots i\left(\sigma_{s_{3}-3}\right)\right\}
\end{aligned}
$$

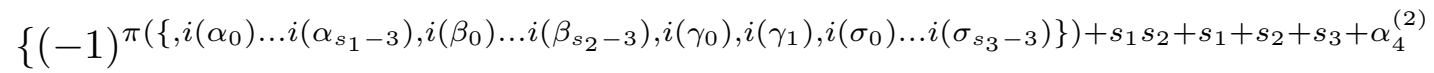

$$
\begin{aligned}
& \left(1+i\left(\gamma_{0}\right)\right) !\left(2+i\left(\gamma_{1}\right)\right) ! \prod_{k_{1}=1}^{s_{1}-2}\left(k_{1}+i\left(\alpha_{k_{1}}-1\right)\right) ! \\
& \prod_{k_{2}=1}^{s_{2}-2}\left(k_{2}+i\left(\beta_{k_{2}}-1\right)\right) ! \prod_{k_{3}=1}^{s_{3}-2}\left(k_{3}+i\left(\beta_{k_{3}}-1\right)\right) ! \\
& \times \prod_{j=1}^{3} \frac{\left(\left(2 s_{j}-1\right) !\right)^{2}}{\alpha_{j} ! \beta_{j} !\left(2 s_{j}-1-\alpha_{j}\right) !\left(2 s_{j}-1-\beta_{j}\right) !} \\
& \times\left.\frac{\Gamma(3-2 u) \Gamma(1+2 u-2 \Sigma s)}{\Gamma\left(3-2 u-\alpha_{4}^{(1)}\right) \Gamma\left(1+2 u-2 \Sigma s-\beta_{4}^{(1)}\right)}\right|_{u \rightarrow 4} \\
& \times \frac{\left(s_{1}-1\right) !\left(s_{2}-1\right) !\left(s_{3}-1\right) !\left(\sum s-3\right) !}{\prod_{i=1}^{4} \prod_{j=2 ; j>i}^{5} R_{i j} ! \prod_{k=1}^{5} \prod_{l=1 ; k \neq l}^{5} Q_{k l} !} \\
& (i)^{\sum_{i, j=1 ; i \neq j}^{5} Q_{i j}}(-1)^{\sum_{j=1}^{5}\left(Q_{1 j}+Q_{2 j}\right)-Q_{21}+Q_{43}+Q_{43}+Q_{45}+Q_{53}}
\end{aligned}
$$

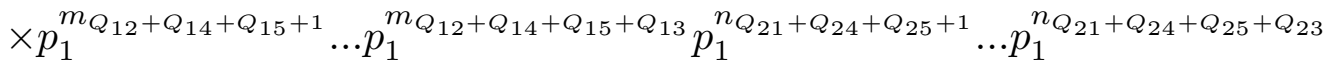

$$
\begin{aligned}
& p_{1}^{q_{Q_{41}+Q_{42}+Q_{45}+1}} \ldots p_{1}^{q_{Q_{41}+Q_{42}+Q_{45}+Q_{43}}} p_{1}^{r_{Q_{51}+Q_{52}+Q_{54}+1}} \ldots p_{1}^{r_{Q_{51}+Q_{52}+Q_{54}+Q_{53}}} \\
& p_{2}^{m_{Q_{12}+Q_{14}+1}} \ldots p_{2}^{m_{Q_{12}}+Q_{14}+Q_{15}} p_{2}^{n_{Q_{21}+Q_{24}+1}} \ldots p_{2}^{n_{Q_{21}+Q_{24}+Q_{25}}} \\
& p_{2}^{q_{Q_{41}+Q_{42}+1}} \ldots p_{2}^{q_{Q_{41}+Q_{42}+Q_{45}}} p_{2}^{t_{Q_{31}+Q_{32}+Q_{34}+1}} \ldots p_{2}^{t_{Q_{31}+Q_{32}+Q_{34}+Q_{35}}} \\
& p_{3}^{m_{Q_{12}+1}} \ldots p_{3}^{m_{Q_{12}+Q_{14}}} p_{3}^{n_{Q_{21}+1}} \ldots p_{3}^{n_{Q_{21}+Q_{24}}} \\
& p_{3}^{r_{Q_{51}+Q_{52}+1}} \ldots p_{3}^{r_{Q_{51}+Q_{52}+Q_{54}}} p_{3}^{t_{Q_{31}+Q_{32}+1}} \ldots p_{3}^{t_{Q_{31}+Q_{32}+Q_{34}}}
\end{aligned}
$$




$$
\begin{aligned}
& \times p_{4}^{m_{1}} \ldots p_{4}^{m_{Q_{12}}} p_{4}^{q_{Q_{41}+1}} \ldots p_{4}^{q_{Q_{41}+Q_{42}}} p_{4}^{r_{Q_{51}+1}} \ldots p_{4}^{r_{Q_{51}+Q_{52}}} p_{4}^{t_{Q_{31}+1}} \ldots p_{4}^{t_{Q_{31}+Q_{32}}} \\
& p_{5}^{n_{1}} \ldots p_{5}^{n_{Q_{21}}} p_{5}^{q_{1}} \ldots p_{5}^{q_{Q_{41}}} p_{5}^{r_{1}} \ldots p_{5}^{r_{Q_{51}}} p_{5}^{t_{1}} \ldots p_{5}^{t_{Q_{31}}} \\
& \times \frac{\Gamma\left[p_{2} p_{3}+9-T_{45}-2 \Sigma s-\beta_{2}-\frac{1}{2}\left(s_{2}-2\right)^{2}-\sum_{k=1}^{s_{2}-2} i\left(\beta_{k-1}\right)\right]}{\Gamma\left[-p_{2} p_{4}+T_{25}-s_{2}(\Sigma s-4)+\frac{1}{2}\left(s_{2}-2\right)^{2}+\beta_{2}+\sum_{k=1}^{s_{2}-2} i\left(\beta_{k-1}\right)\right]} \\
& \times \frac{\Gamma\left[p_{3} p_{4}-T_{24}+2 s_{2}-\alpha_{2}\right]}{\sin \left[\pi\left(p_{2} p_{4}-T_{25}+s_{2}(\Sigma s-4)-\frac{1}{2}\left(s_{2}-2\right)^{2}-\beta_{2}-\sum_{k=1}^{s_{2}-2} i\left(\beta_{k-1}\right)\right)\right]} \\
& \times \Gamma\left[p_{2} p_{4}+p_{2} p_{3}+p_{1} p_{3}+6-T_{25}-T_{45}-T_{34}+\right. \\
& \left.\left(s_{2}-2\right)(\Sigma s-4)-\frac{1}{2}\left(s_{2}-2\right)^{2}+2 s_{3}-\alpha_{3}-\sum_{k=1}^{s_{2}-2} i\left(\beta_{k-1}\right)-i\left(\gamma_{0}\right)-i\left(\gamma_{1}\right)\right] \\
& \Gamma^{-1}\left[p_{2} p_{4}+p_{2} p_{3}+2-T_{25}-T_{45}+\left(s_{2}-2\right)(\Sigma s-4)\right. \\
& \left.-\frac{1}{2}\left(s_{2}-2\right)^{2}-\sum_{k=1}^{s_{2}-2} i\left(\beta_{k-1}\right)-i\left(\gamma_{0}\right)-i\left(\gamma_{1}\right)\right] \\
& \times \Gamma\left[p_{2} p_{4}+p_{2} p_{3}+p_{1} p_{3}+p_{3} p_{4}+6-T_{24}+2 s_{2}-\alpha_{2}-T_{25}-T_{45}-T_{34}\right. \\
& \left.+\left(s_{2}-2\right)(\Sigma s-4)-\frac{1}{2}\left(s_{2}-2\right)^{2}+2 s_{3}-\alpha_{3}-\sum_{k=1}^{s_{2}-2} i\left(\beta_{k-1}\right)-i\left(\gamma_{0}\right)-i\left(\gamma_{1}\right)\right] \\
& \times_{3} F_{2}\left[-p_{1} p_{2}+T_{35}-\left(s_{3}+2\right)(\Sigma s-4)+\frac{1}{2}\left(s_{3}-2\right)^{2}+\beta_{3}+\sum_{k=1}^{s_{3}-2} i\left(\beta_{k-1}\right)\right. \\
& p_{2} p_{4}+1-T_{25}+s_{2}(\Sigma s-4)-\frac{1}{2}\left(s_{2}-2\right)^{2}-\beta_{2}-\sum_{k=1}^{s_{2}-2} i\left(\beta_{k-1}\right) ; \\
& p_{2} p_{4}+p_{2} p_{3}+p_{1} p_{3}+6-T_{25}-T_{45}-T_{34}+\left(s_{2}-2\right)(\Sigma s-4) \\
& -\frac{1}{2}\left(s_{2}-2\right)^{2}+2 s_{3}-\alpha_{3}-\sum_{k=1}^{s_{2}-2} i\left(\beta_{k-1}\right)-i\left(\gamma_{0}\right)-i\left(\gamma_{1}\right) \\
& p_{2} p_{4}+p_{2} p_{3}+2-T_{25}-T_{45}+\left(s_{2}-2\right)(\Sigma s-4) \\
& -\frac{1}{2}\left(s_{2}-2\right)^{2}-\sum_{k=1}^{s_{2}-2} i\left(\beta_{k-1}\right)-i\left(\gamma_{0}\right)-i\left(\gamma_{1}\right) \\
& p_{2} p_{4}+p_{2} p_{3}+p_{1} p_{3}+p_{3} p_{4}+6-T_{25}-T_{45}-T_{34}-T_{24}+\left(s_{2}-2\right)(\Sigma s-4) \\
& \left.-\frac{1}{2}\left(s_{2}-2\right)^{2}+2 s_{2}+2 s_{3}-\alpha_{2}-\alpha_{3}-\sum_{k=1}^{s_{2}-2} i\left(\beta_{k-1}\right)-i\left(\gamma_{0}\right)-i\left(\gamma_{1}\right) ; 1\right]
\end{aligned}
$$




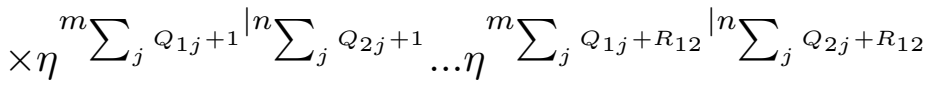

$$
\begin{aligned}
& \eta^{m} \sum_{j} Q_{1 j}+1+R_{12}{ }^{\mid q} \sum_{j}{ }^{Q_{4 j}+1} \ldots \eta^{m} \sum_{j} Q_{1 j}+R_{12}+R_{14}{ }^{\mid q} \sum_{j} Q_{4 j}+R_{14} \\
& \eta^{m} \sum_{j} Q_{1 j}+R_{12}+R_{14}+1^{\mid r} \sum_{j} Q_{5 j}+1 \ldots \eta^{m} \sum_{j} Q_{1 j}+R_{12}+R_{14}+R_{15}{ }^{\mid r} \sum_{j} Q_{5 j}+R_{15}
\end{aligned}
$$

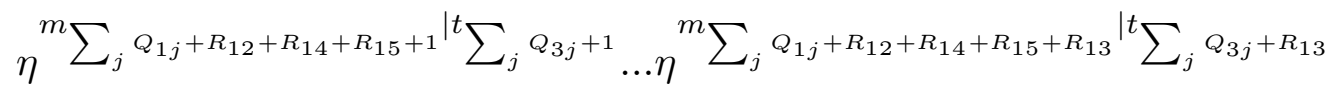

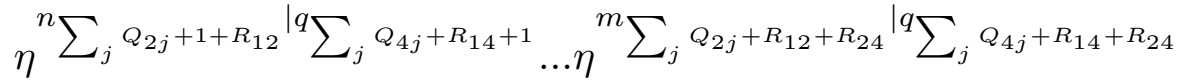

$$
\begin{aligned}
& \eta^{n} \sum_{j} Q_{2 j}+1+R_{12}+R_{24}{ }^{\mid r} \sum_{j} Q_{5 j}+R_{15}+1 \ldots \eta^{m} \sum_{j} Q_{2 j}+R_{12}+R_{24}+R_{25}{ }^{\mid r} \sum_{j} Q_{5 j}+R_{15}+R_{25}
\end{aligned}
$$

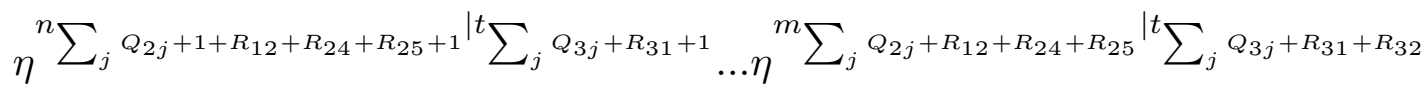

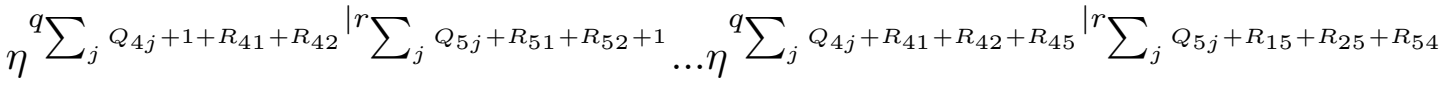

$$
\begin{aligned}
& \eta^{q} \sum_{j}{ }^{Q_{4 j}+1+R_{41}+R_{42}+R_{45}{ }^{\mid t} \sum_{j} Q_{3 j}+R_{31}+R_{32}+1} \ldots \eta^{q} \sum_{j} Q_{4 j}+R_{41}+R_{42}+R_{45}+R_{43} \mid t \sum_{j} Q_{3 j}+R_{31}+R_{32}+R_{34}
\end{aligned}
$$

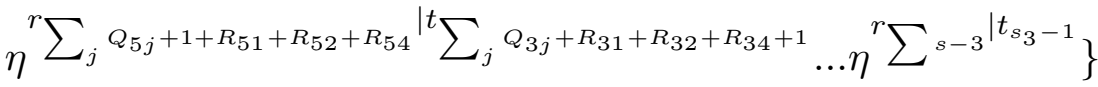

where

$$
T_{i j}=2 R_{i j}+Q_{i j}+Q_{j i}
$$

and the additional constraint is imposed on the partitions:

$$
\sum_{j=2}^{5} Q_{j 1}+\sum_{k=1}^{s_{1}-2} i\left(\alpha_{k-1}\right)=3+\frac{s_{1}\left(s_{1}-1\right)}{2}
$$

stemming from the fact that only $w^{0}$-terms conmtribute to the amplitude, with all others vanishing in the limit $w \rightarrow \infty$. This concludes the computation of the five-point amplitude for the localized quintic interaction. In the next section we shall discuss the construction of the quintic higher spin vertex, related to this amplitude.

\section{Reading off the Quintic Vertex}

In the previous section we have computed the five-point worldsheet amplitude, related to the interaction of masssless higher spin in the quintic order. By itself, this amplitude does not describe yet the 5-point interaction vertex in the low energy effective action: it is only gauge-invariant under gauge (BRST) transformations at the linearized level. To read off the interaction vertex with the full gauge symmetry, one has to subtract from it the 
terms, produced as a result of the worldsheet RG flows of the effective action's terms at lower orders, such as cubic and quartic. Generally speaking, there are two possible sources of such terms at the quintic order: the flow of the quartic vertex in the leading $\alpha^{\prime}$ order and the flow of the cubic vertex in the subleading $\alpha^{\prime}$ order. In general, computation of these flow terms would be quite complicated; in particular they would involve the flows stemming from all the diversity of the quartic vertices which by themselves are tedious and complex. Moreover, since the quartic interactions are generally nonlocal, one would generally expect the flow terms to retain these nonlocalities, destroying the local structure of the amplitude (35)-(37). Fortunately, however, for the spin combinations considered in our work things again get drastically simplified: as we pointed out above, the fourpoint correlation functions contributing to the worldsheet $\beta$-function of the space-time frame-like field with the highest spin value, relevant to the flow terms at the quintic order, vanish identically, as it is impossible to accommodate the full contractions of the $\psi$-fermions consistently with the ghost number balance restrictions. Therefore the quartic interactions, relevant to the flow terms at the quintic order, stem themselves from the flows from the previous (cubic) order. For this reason, the flow contributions are reduced to the double composition of the RG flows of the cubic terms which structure is relatively simple to control. As the structure of the cubic higher spin vertices is determined by the structure constants of the higher spin algebra [44], [45] and the quintic vertex is cubic in the structure constants, the effect of the cubic terms flows can be expressed by regularizing the poles in $s_{i j}$-channels present in the amplitude (35)-(37) due to the Euler's gamma-functions and the hypergeometric function where

$$
\begin{aligned}
& s_{12}=\frac{1}{2}\left(p_{1}-p_{2}\right)^{2} ; s_{13}=\frac{1}{2}\left(p_{1}-p_{3}\right)^{2} ; s_{14}=\frac{1}{2}\left(p_{1}-p_{4}\right)^{2} \\
& s_{23}=\frac{1}{2}\left(p_{2}-p_{3}\right)^{2} ; s_{24}=\frac{1}{2}\left(p_{2}-p_{4}\right)^{2} ; s_{34}=\frac{1}{2}\left(p_{3}+p_{4}\right)^{2} \\
& \sum_{i, j} s_{i j}=0
\end{aligned}
$$

are the generalized Mandelstam variables. Subtracting the poles resulting from the flows of the cubic vertices using the procedure similar to those explained in 449], [46] and taking the field theory limit we find the quintic vertex stemming from the $\beta$-function of the highest 
spin field is given by:

$$
\begin{aligned}
& A\left(p_{1}, \ldots, p_{5}\right)=6 \pi \Omega_{m_{1} \ldots m_{s_{1}-1} \mid \lambda_{0} \ldots \lambda_{s_{1}-3}}\left(p_{5}\right) \Omega_{n_{1} \ldots n_{s_{2}-1} \mid \lambda_{s_{1}-2 \ldots} \ldots \lambda_{s_{1}+s_{2}-5}}\left(p_{4}\right) \\
& \Omega_{q_{1} q_{2} q_{3} \mid \lambda_{s_{1}+s_{2}-4} \lambda_{s_{1}+s_{2}-3}}\left(p_{3}\right) \Omega_{r_{1} \ldots r_{\Sigma s-3}}^{\lambda_{0} \ldots \lambda_{\Sigma s-5}}\left(p_{2}\right) \Omega_{t_{1} \ldots t_{s_{3}-1} \mid \lambda_{s_{1}+s_{2}-2 \ldots \lambda \lambda_{\Sigma s-5}}}\left(p_{1}\right)
\end{aligned}
$$

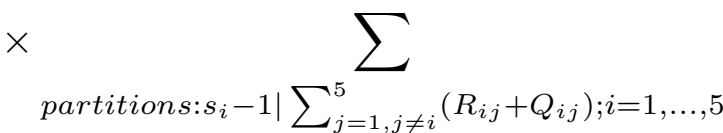

$$
\begin{aligned}
& \sum \\
& \text { partitions: } 2(u-2) \equiv 4 \mid \alpha_{1}+\alpha_{2}+\alpha_{3}+\alpha_{4}^{(1)}+\alpha_{4}^{(2)} \\
& \sum \\
& \text { partitions: } 2(\Sigma s-u) \equiv 2(\Sigma s-4) \mid \beta_{1}+\beta_{2}+\beta_{3}+\beta_{4}^{(1)}+\beta_{4}^{(2)} \\
& \sum_{\text {permutations: }\left\{, i\left(\alpha_{0}\right) \ldots i\left(\alpha_{s_{1}-3}\right), i\left(\beta_{0}\right) \ldots i\left(\beta_{s_{2}-3}\right), i\left(\gamma_{0}\right), i\left(\gamma_{1}\right), i\left(\sigma_{0}\right) \ldots i\left(\sigma_{s_{3}-3}\right)\right\}}
\end{aligned}
$$

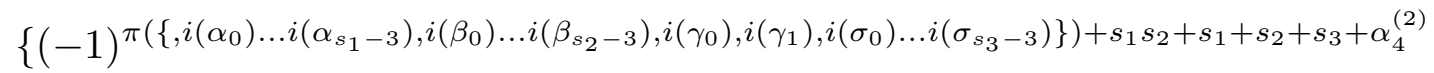

$$
\begin{aligned}
& \left(1+i\left(\gamma_{0}\right)\right) !\left(2+i\left(\gamma_{1}\right)\right) ! \prod_{k_{1}=1}^{s_{1}-2}\left(k_{1}+i\left(\alpha_{k_{1}}-1\right)\right) ! \\
& \prod_{k_{2}=1}^{s_{2}-2}\left(k_{2}+i\left(\beta_{k_{2}}-1\right)\right) ! \prod_{k_{3}=1}^{s_{3}-2}\left(k_{3}+i\left(\beta_{k_{3}}-1\right)\right) ! \\
& \times \prod_{j=1}^{3} \frac{\left(\left(2 s_{j}-1\right) !\right)^{2}}{\alpha_{j} ! \beta_{j} !\left(2 s_{j}-1-\alpha_{j}\right) !\left(2 s_{j}-1-\beta_{j}\right) !} \\
& \times\left.\frac{\Gamma(3-2 u) \Gamma(1+2 u-2 \Sigma s)}{\Gamma\left(3-2 u-\alpha_{4}^{(1)}\right) \Gamma\left(1+2 u-2 \Sigma s-\beta_{4}^{(1)}\right)}\right|_{u \rightarrow 4} \\
& \times \frac{\left(s_{1}-1\right) !\left(s_{2}-1\right) !\left(s_{3}-1\right) !\left(\sum s-3\right) !}{\prod_{i=1}^{4} \prod_{j=2 ; j>i}^{5} R_{i j} ! \prod_{k=1}^{5} \prod_{l=1 ; k \neq l}^{5} Q_{k l} !} \\
& (i)^{\sum_{i, j=1 ; i \neq j}^{5} Q_{i j}}(-1)^{\sum_{j=1}^{5}\left(Q_{1 j}+Q_{2 j}\right)-Q_{21}+Q_{43}+Q_{43}+Q_{45}+Q_{53}} \\
& \times p_{1}^{m_{Q_{12}}+Q_{14}+Q_{15}+1} \ldots p_{1}^{m_{Q_{12}+Q_{14}+Q_{15}+Q_{13}}} p_{1}^{n_{Q_{21}+Q_{24}+Q_{25}+1}} \ldots p_{1}^{n_{Q_{21}+Q_{24}+Q_{25}+Q_{23}}} \\
& p_{1}^{q_{Q_{41}+Q_{42}+Q_{45}+1}} \ldots p_{1}^{q_{Q_{41}+Q_{42}+Q_{45}+Q_{43}}} p_{1}^{r_{Q_{51}+Q_{52}+Q_{54}+1}} \ldots p_{1}^{r_{Q_{51}+Q_{52}+Q_{54}+Q_{53}}} \\
& p_{2}^{m_{Q_{12}+Q_{14}+1}} \ldots p_{2}^{m_{Q_{12}+Q_{14}+Q_{15}}} p_{2}^{n_{Q_{21}+Q_{24}+1}} \ldots p_{2}^{n_{Q_{21}+Q_{24}+Q_{25}}} \\
& p_{2}^{q_{Q_{41}+Q_{42}+1}} \ldots p_{2}^{q_{Q_{41}+Q_{42}+Q_{45}}} p_{2}^{t_{Q_{31}+Q_{32}+Q_{34}+1}} \ldots p_{2}^{t_{Q_{31}+Q_{32}+Q_{34}+Q_{35}}} \\
& p_{3}^{m_{Q_{12}+1}} \ldots p_{3}^{m_{Q_{12}+Q_{14}}} p_{3}^{n_{Q_{21}+1}} \ldots p_{3}^{n_{Q_{21}+Q_{24}}} \\
& p_{3}^{r_{Q_{51}+Q_{52}+1}} \ldots p_{3}^{r_{Q_{51}+Q_{52}+Q_{54}}} p_{3}^{t_{Q_{31}+Q_{32}+1}} \ldots p_{3}^{t_{Q_{31}+Q_{32}+Q_{34}}}
\end{aligned}
$$




$$
\begin{aligned}
& \times p_{4}^{m_{1}} \ldots p_{4}^{m_{Q_{12}}} p_{4}^{q_{Q_{41}+1}} \ldots p_{4}^{q_{Q_{41}+Q_{42}}} p_{4}^{r_{Q_{51}+1}} \ldots p_{4}^{r_{Q_{51}+Q_{52}}} p_{4}^{t_{Q_{31}+1}} \ldots p_{4}^{t_{Q_{31}+Q_{32}}} \\
& p_{5}^{n_{1}} \ldots p_{5}^{n_{Q_{21}}} p_{5}^{q_{1}} \ldots p_{5}^{q_{Q_{41}}} p_{5}^{r_{1}} \ldots p_{5}^{r_{Q_{51}}} p_{5}^{t_{1}} \ldots p_{5}^{t_{Q_{31}}} \\
& \times\left[\left(-9+T_{45}+2 \Sigma s+\beta_{2}+\frac{1}{2}\left(s_{2}-2\right)^{2}+\sum_{k=1}^{s_{2}-2} i\left(\beta_{k-1}\right)\right) !\right]^{-1} \\
& \times L\left(-9+T_{45}+2 \Sigma s+\beta_{2}+\frac{1}{2}\left(s_{2}-2\right)^{2}+\sum_{k=1}^{s_{2}-2} i\left(\beta_{k-1}\right)\right) \\
& \times\left(-T_{25}+s_{2}(\Sigma s-4)-\frac{1}{2}\left(s_{2}-2\right)^{2}-\beta_{2}-\sum_{k=1}^{s_{2}-2} i\left(\beta_{k-1}\right)\right) !\left(1-T_{24}+2 s_{2}-\alpha_{2}\right) ! \\
& \times\left[5-T_{25}-T_{45}-T_{34}+\left(s_{2}-2\right)(\Sigma s-4)-\frac{1}{2}\left(s_{2}-2\right)^{2}\right. \\
& \left.+2 s_{3}-\alpha_{3}-\sum_{k=1}^{s_{2}-2} i\left(\beta_{k-1}\right)-i\left(\gamma_{0}\right)-i\left(\gamma_{1}\right)\right] \text { ! } \\
& {\left[\left(1-T_{25}-T_{45}+\left(s_{2}-2\right)(\Sigma s-4)-\frac{1}{2}\left(s_{2}-2\right)^{2}-\sum_{k=1}^{s_{2}-2} i\left(\beta_{k-1}\right)-i\left(\gamma_{0}\right)-i\left(\gamma_{1}\right)\right) !\right]^{-1}} \\
& \times\left[5-T_{24}+2 s_{2}-\alpha_{2}-T_{25}-T_{45}-T_{34}+\left(s_{2}-2\right)(\Sigma s-4)-\frac{1}{2}\left(s_{2}-2\right)^{2}\right. \\
& \left.+2 s_{3}-\alpha_{3}-\sum_{k=1}^{s_{2}-2} i\left(\beta_{k-1}\right)-i\left(\gamma_{0}\right)-i\left(\gamma_{1}\right)\right] \text { ! } \\
& \times_{3} F_{2}\left[T_{35}-\left(s_{3}+2\right)(\Sigma s-4)+\frac{1}{2}\left(s_{3}-2\right)^{2}+\beta_{3}+\sum_{k=1}^{s_{3}-2} i\left(\beta_{k-1}\right)\right. \\
& 1-T_{25}+s_{2}(\Sigma s-4)-\frac{1}{2}\left(s_{2}-2\right)^{2}-\beta_{2}-\sum_{k=1}^{s_{2}-2} i\left(\beta_{k-1}\right) \\
& 6-T_{25}-T_{45}-T_{34}+\left(s_{2}-2\right)(\Sigma s-4)-\frac{1}{2}\left(s_{2}-2\right)^{2} \\
& +2 s_{3}-\alpha_{3}-\sum_{k=1}^{s_{2}-2} i\left(\beta_{k-1}\right)-i\left(\gamma_{0}\right)-i\left(\gamma_{1}\right) \\
& 2-T_{25}-T_{45}+\left(s_{2}-2\right)(\Sigma s-4)-\frac{1}{2}\left(s_{2}-2\right)^{2}-\sum_{k=1}^{s_{2}-2} i\left(\beta_{k-1}\right)-i\left(\gamma_{0}\right)-i\left(\gamma_{1}\right) \\
& 6-T_{25}-T_{45}-T_{34}-T_{24}+\left(s_{2}-2\right)(\Sigma s-4)-\frac{1}{2}\left(s_{2}-2\right)^{2}+2 s_{2}+2 s_{3} \\
& \left.-\alpha_{2}-\alpha_{3}-\sum_{k=1}^{s_{2}-2} i\left(\beta_{k-1}\right)-i\left(\gamma_{0}\right)-i\left(\gamma_{1}\right) ; 1\right]
\end{aligned}
$$




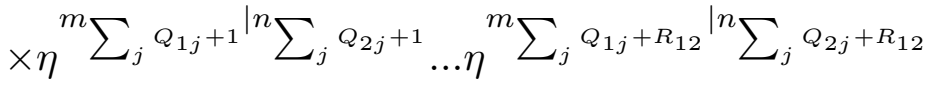

$$
\begin{aligned}
& \eta^{m} \sum_{j} Q_{1 j}+1+R_{12}{ }^{\mid q} \sum_{j}{ }^{Q_{4 j}+1} \ldots \eta^{m} \sum_{j} Q_{1 j}+R_{12}+R_{14}{ }^{\mid q} \sum_{j} Q_{4 j}+R_{14}
\end{aligned}
$$

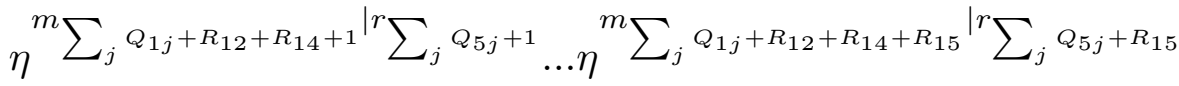

$$
\begin{aligned}
& \eta^{m} \sum_{j} Q_{1 j}+R_{12}+R_{14}+R_{15}+1{ }^{\mid t} \sum_{j} Q_{3 j}+1 \ldots \eta^{m} \sum_{j} Q_{1 j}+R_{12}+R_{14}+R_{15}+R_{13}{ }^{\mid t} \sum_{j} Q_{3 j}+R_{13}
\end{aligned}
$$

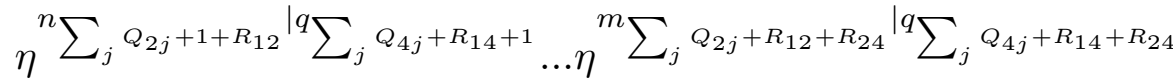

$$
\begin{aligned}
& \eta^{n} \sum_{j} Q_{2 j}+1+R_{12}+R_{24}{ }^{\mid r} \sum_{j} Q_{5 j}+R_{15}+1 \ldots \eta^{m} \sum_{j} Q_{2 j}+R_{12}+R_{24}+R_{25}{ }^{\mid r} \sum_{j} Q_{5 j}+R_{15}+R_{25}
\end{aligned}
$$

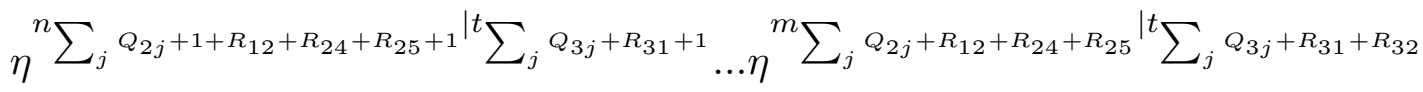

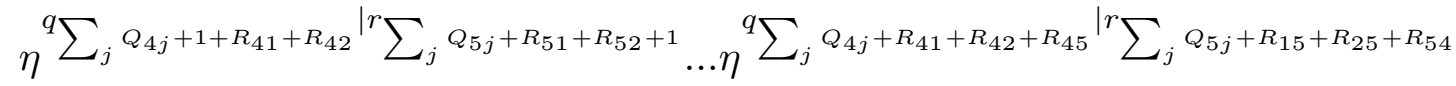

$$
\begin{aligned}
& \eta^{q} \sum_{j} Q_{4 j}+1+R_{41}+R_{42}+R_{45}\left|t \sum_{j} Q_{3 j}+R_{31}+R_{32}+1 \ldots \eta^{q} \sum_{j} Q_{4 j}+R_{41}+R_{42}+R_{45}+R_{43}\right| t \sum_{j} Q_{3 j}+R_{31}+R_{32}+R_{34}
\end{aligned}
$$

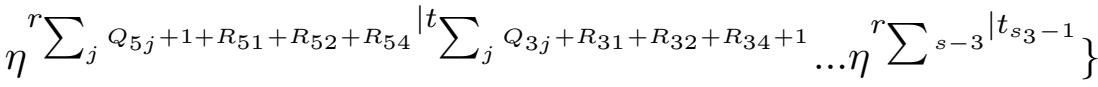

where

$$
L(n)=\sum_{m=1}^{n} \frac{1}{m}
$$

This concludes the evaluation of the quintic interaction vertex.

\section{Conclusion}

In this work we have considered a very special limit of higher spin quintic interaction, limited to the case when the sum of three spins participating in the interaction roughly equals the sum of the remaining two, or which one must be small anough and another must be large. In this case, the ghost structure of the operators drastically simplifies the calculations and the absence of $\mathrm{RG}$ flows from the quartic order, contributing at the fifth order, makes the construction of the interaction 5-vertex from the scattering amplitude a relatively straightforward procedure. Despite the specific choice of the spin values considered in this work, it is remarkable that the structure of the 5-vertex can be extracted from string theory in the low energy limit. One particularly important result is the locality of the quintic amplitude in this limit. This is the intriguing novelty of the quintic interaction all the known examples of the vertices at the previous (quartic) order are essentially nonlocal, and the vertex (41)-(43) constructed in this paper has no quartic 
analogue (which vanishes by the ghost/ $\psi$-number constraints). This may bear important important implications for the higher spin holography, as the nonlocality of higher spin vertices versus their local counterparts in the dual CFT's is the well-known puzzle [50], [51], [52], [53], [54]. Despite the vanishing of the four-point analogue of the amplitude considered in this work, one may still hope to find alternative mechanisms for localization at the quartic level, at least in the AdS space. Our hope is that the string theory may provide efficient and powerful tools to address these issues, as well as to approach the higher orders of higher spin interactions, at least for specific spin values. It is also of interest to find the holographic interpretation of the localization effect, pointed out in our calculations. For that, it is on the other hand necessary to have better understanding of the higher-spin nonlocalities from the string theory side. So far, string theory has been able to account for very limited and simplistic types of higher spin nonlocalities only. For that, the class of $V_{s-1 \mid s-3}$-operators considered in this work, may not be sufficient and one may need to find explicit solutions of the operator equations (15)-(16) for the generalized zero torsion constraints. These constraints are generally hard to work with in the formalism of the on-shell (first-quantized) string theory. Alternatively, in the string field theory (SFT) approach the nonlocalities may be naturally encripted in the structure of operators in the cohomologies of the BRST charge shifted by the appropriate analytic solutions in open string field theory [55]. The obvious advantage of this approach is the background independence, which in theory may allow us to penetrate beyond the realm of standard string perturbation theory. Although at this time our understanding of how the analytic solutions work to describe higher spin interaction is still very preliminary and limited, in the end it seems plausible that the language of shifted BRST cohomologies in open string field theory may be the most natural and efficient to understand the nonlocalities in higher spin interactions.

\section{Acknowledgements}

It is a pleasure to thank Lars Brink and other organizers and participants of the International Workshop on Higher Spin Gauge Theories at Nanyang Technological University, Singapore in November, 2015 for hospitality and inspiring discussions. This work is supported by the National Natural Science Foundation of China under grant 11575119. 


\section{References}

[1] C. Fronsdal, Phys. Rev. D18 (1978) 3624

[2] A. Sagnotti, E. Sezgin, P. Sundell, hep-th/0501156

[3] D. Francia, A. Sagnotti, Phys. Lett. B53 (2002) 303

[4] D. Francia, A. Sagnotti, Class. Quant. Grav. 20 (2003) S473

[5] D. Francia, J. Mourad, A. Sagnotti, Nucl. Phys. B773 (2007) 203

[6] D. Sorokin, AIP Conf. Proc. 767, 172 (2005)

[7] M. Vasiliev, Phys. Lett. B243 (1990) 378

[8] M. Vasiliev, Int. J. Mod. Phys. D5 (1996) 763

[9] M. Vasiliev, Phys. Lett. B567 (2003) 139

[10] D.S. Ponomarev, M.A. Vasiliev, Nucl.Phys.B839:466-498,2010

[11] S. Deser, Z. Yang, Class. Quant. Grav 7 (1990) 1491

[12] A. Bengtsson, I. Bengtsson, N. Linden, Class. Quant. Grav. 4 (1987) 1333

[13] W. Siegel, B. Zwiebach, Nucl. Phys. B282 (1987) 125

[14] W. Siegel, Nucl. Phys. B 263 (1986) 93

[15] A. Neveu, H. Nicolai, P. West, Nucl. Phys. B264 (1986) 573

[16] T. Damour, S. Deser, Ann. Poincare Phys. Theor. 47 (1987) 277

[17] A. Bengtsson, I. Bengtsson, L. Brink, Nucl. Phys. B227 (1983) 31

[18] X. Bekaert, N. Boulanger, S. Cnockaert, J. Math. Phys 46 (2005) 012303

[19] J. Labastida, Nucl. Phys. B322 (1989)

[20] J. Labastida, Phys. rev. Lett. 58 (1987) 632

[21] L. Brink, R.Metsaev, M. Vasiliev, Nucl. Phys. B 586 (2000)183

[22] X. Bekaert, S. Cnockaert, C. Iazeolla, M.A. Vasiliev, IHES-P-04-47, ULB-TH-04-26, ROM2F-04-29, FIAN-TD-17-04, Sep 2005 86pp.

[23] A. Fotopoulos, M. Tsulaia, Phys.Rev.D76:025014,2007

[24] I. Buchbinder, A. Fotopoulos, A. Petkou, Phys.Rev.D74:105018,2006

[25] G. Bonelli, Nucl.Phys.B 669 (2003) 159

[26] I. Buchbinder, A. Pashnev, M. Tsulaia, arXiv:hep-th/0109067

[27] X. Bekaert, N. Boulanger, P. Sundell, arXiv:1007.0435

[28] N. Boulanger, C. Iazeolla, P. Sundell, JHEP 0907 (2009) 013

[29] N. Boulanger, C. Iazeolla, P. Sundell, JHEP 0907 (2009) 014

[30] A. Sagnotti, M. Taronna, arXiv:1006.5242, Nucl.Phys.B842:299-361,2011

[31] M. Taronna, arXiv:1005.3061

[32] Yu. Zinoviev, arXiv:1007.0158

[33] A. Fotopoulos, M. Tsulaia, arXiv:1007.0747

[34] A. Fotopoulos, M. Tsulaia, arXiv:1009.0727

[35] M. Bianchi, J.F. Morales and H. Samtleben, JHEP 0307 (2003) 062

[36] S. Giombi, Xi Yin, arXiv:0912.5105 
[37] S. Giombi, Xi Yin, arXiv:1004.3736

[38] E. Sezgin and P. Sundell, Nucl.Phys.B644:303- 370,2002

[39] B. Sundborg, ucl.Phys.Proc.Suppl. 102 (2001)

[40] N. Boulanger,S. Leclercq, P. Sundell, JHEP 0808(2008) 056

[41] E.D. Skvortsov, Yu.M. Zinoviev, arXiv:1007.4944

[42] R. Manvelyan, K. Mkrtchyan, W. Ruehl, arXiv:1009.1054

[43] R. Manvelyan, K. Mkrtchyan, W. Ruehl, Nucl.Phys.B836:204-221,2010

[44] D. Polyakov, Phys.Rev. D84 (2011) 126004

[45] D. Polyakov, Phys.Rev. D89 (2014) 2, 026010

[46] D. Polyakov, Phys.Rev. D83 (2011) 046005

[47] D. Polyakov, arXiv:1507.06226, to appear in Phys.Rev. D

[48] D. Polyakov,Phys.Rev.D82:066005,2010

[49] I. Klebanov, A Tseytlin, Nucl.Phys.B546:155-181,1999

[50] A. Jevicki, K. Jin, Q. Ye, J.Phys. A46 (2013) 214005

[51] A. Jevicki, K. Jin, Q. Ye, J.Phys. A44 (2011) 465402

[52] X. Bekaert, J. Erdmenger, D. Ponomarev, C. Sleight, arXiv: 1508.04292

[53] X. Bekaert, J. Erdmenger, D. Ponomarev, C. Sleight, JHEP 1503 (2015) 170

[54] R. R. Metsaev, J.Phys. A46 (2013) 214021

[55] M. Schnabl, Adv.Theor.Math.Phys. 10 (2006) 433-501 